\title{
CAMBIOS RESIDENCIALES Y MIGRATORIOS DE LA POBLACIÓN EXTRANJERA EN LA CIUDAD DE VALENCIA (2014-2017)
}

\author{
Julia Salom-Carrasco \\ Instituto Interuniversitario de Desarrollo Local, Universitat de Valencia \\ Julia.Salom@uv.es \\ ORCID iD: http://orcid.org/0000-0001-6436-9761
}

Recibido: 28/03/2021; Aceptado: 20/11/2021; Publicado: 22/12/2021.

Cómo citar este artículo/citation: Salom-Carrasco, Julia (2021). Cambios residenciales y migratorios de la población extranjera en la ciudad de Valencia (2014-2017). Estudios Geográficos, 82 (291), e085. https://doi.org/10.3989/estgeogr.202196.096

\begin{abstract}
RESUMEN: Desde comienzos de siglo se ha producido en las ciudades españolas un importante aumento de la entrada de población extranjera que ha transformado el paisaje social de la ciudad, dando lugar en algunos casos a concentraciones espaciales de este tipo de población en áreas concretas. En este artículo se analizan las tendencias actuales (post-crisis) de localización de la población extranjera en la ciudad central metropolitana, utilizando como caso de estudio la ciudad de Valencia. Para ello se estudian los cambios residenciales y migratorios a escala de sección censal de la población extranjera clasificada por nacionalidad y nivel educativo, aplicando herramientas estadísticas (I Global de Moran y Hot-Spot Analysis) que permiten identificar las tendencias espaciales significativas. Los resultados indican que la movilidad residencial y migratoria de la población extranjera está contribuyendo, en líneas generales, a la consolidación y ampliación geográfica del patrón territorial existente. Sin embargo, se detectan algunos cambios debidos a la emergencia de nuevos espacios atractivos para la población extranjera de nivel educativo más elevado, especialmente nacionales de países de la Unión Europea, en entornos específicos que han sido revitalizados por proyectos urbanísticos concretos o por procesos de renovación urbana más amplios.
\end{abstract}

PALABRAS CLAVE: Cambios residenciales y migratorios, Población extranjera, Hot-Spot Analysis, Valencia.

\section{Residential and migratory changes of the foreign population in the city of Valencia (2014-2017)}

ABSTRACT: Since the beginning of the century, Spanish cities have experienced a significant increase in the influx of foreign population, which has transformed the social landscape of the city, leading in some cases to spatial concentrations of this type of population in specific areas. This article analyses current (post-crisis) trends in the location of the foreign population in the central metropolitan city, using the city of Valencia as a case study. To do so, we study the residential and migratory changes at the census tract level of the foreign population classified by nationality and educational level, applying statistical tools (Moran's Global I and Hot-Spot Analysis) that allow us to identify significant spatial trends. The results indicate that the residential and migratory mobility of the foreign population is contributing, in general terms, to the consolidation and geographical extension of the existing territorial pattern. Some changes are also detected due to the emergence of new attractive spaces for the foreign population with a higher educational level, especially EU nationals, in specific environments that have been revitalised by specific urban development projects or by wider urban renewal processes.

KEYWORDS: Residential and migratory changes, Foreign population, Hot-Spot Analysis, Valencia- Spain. 


\section{INTRODUCCIÓN}

Desde comienzos de siglo se ha producido en las ciudades españolas un importante aumento de la entrada de población extranjera que ha transformado el paisaje social de la ciudad, dando lugar en algunos casos a concentraciones espaciales de este tipo de población que pueden tener consecuencias socialmente negativas (Iglesias Pascual, 2015, 2016; Sorando y Leal, 2019). La crisis económica de 2008 paralizó en gran medida la entrada de inmigrantes, produciéndose además la salida de una gran parte de esta población, que ha regresado a su país o se ha trasladado a otras zonas a la búsqueda de mejores oportunidades de empleo. Sin embargo, las consecuencias espaciales de estas dinámicas sociales sobre el espacio urbano siguen siendo evidentes y, probablemente, condicionan las preferencias residenciales actuales y el patrón territorial del repunte de la inmigración que se produce a partir de 2013. En este artículo analizamos las tendencias actuales (post-crisis) de localización de este tipo de población en la ciudad central metropolitana, utilizando como caso de estudio la ciudad de Valencia. Para ello, analizamos la movilidad residencial y migratoria ${ }^{1}$ de la población extranjera entre enero de 2014 y enero de 2018. Se trata de una etapa que se podría considerar hoy ya prácticamente cerrada, ya que es previsible que el impacto económico y social de la pandemia sobre el crecimiento demográfico urbano abra un nuevo periodo cuyo sentido todavía desconocemos (Florida, Rodríguez-Pose y Storper, 2020).

La relación entre movilidad residencial y migratoria y estructura urbana está claramente establecida desde hace tiempo en el análisis urbano. La movilidad residencial es un factor importante en la configuración de áreas urbanas socialmente diferenciadas, puesto que está condicionada tanto por las necesidades y expectativas de vivienda, a su vez mediatizadas por la renta, el estilo de vida, el estatus familiar y la imagen de la persona que se desplaza, como por la oferta y oportunidades de vivienda que ofrece el mercado inmobiliario (Pacione, 2009, p. 203). Por otra parte, tiene una importante conexión con la movilidad social, ya que los cambios de vivienda implican en cierta manera una reubicación en la escala social (DuqueCalvache, 2017). En el caso español, la entrada de población extranjera producida a partir de principios de siglo y sus posteriores cambios residenciales han tenido un importante efecto sobre la estructura social del espacio urbano. Tal y como apunta Andújar (2017) para el caso de Madrid, durante el periodo 2000-2007 las migraciones y la movilidad residencial de la po- blación extranjera actuaron de forma determinante en los cambios en la composición socioeconómica de las distintas zonas de la ciudad, dando lugar a una creciente especialización socioespacial de las prácticas residenciales. Durante la posterior etapa de crisis (2008-2013) se produce un incremento de la proximidad social de las prácticas territoriales; es decir, los desplazamientos residenciales de los agentes sociales ya no conllevan transformaciones en los barrios que implican, lo que indica que se estarían produciendo entre espacios de una composición social similar. En el caso de la población extranjera, esto significa que, en este periodo, se asienta mayoritariamente en barrios en los que ya existe una proporción elevada de extranjeros o en los que también hay salidas de este colectivo, por lo que no genera trasformaciones con su llegada, consolidándose así la estructura sociodemográfica formada en la etapa anterior (Andújar, 2017).

El objetivo de este artículo es verificar si, en el caso de la ciudad de Valencia, la estructura sociodemográfica del espacio urbano conformada en el periodo de crecimiento migratorio se mantiene y consolida en la etapa post-crisis, o si el repunte de la movilidad de la población extranjera derivada de la recuperación económica ha producido algún cambio que modifique el modelo territorial y, en tal caso, en qué sentido actúa. De forma más específica, pretendemos dar respuesta a las siguientes cuestiones: 1) ¿cuál es el patrón espacial de los cambios residenciales y migratorios de la población extranjera durante el periodo reciente 2014-2018?, 2) ¿es posible identificar espacios con tendencia a la especialización socioresidencial de población extranjera de dentro y fuera de la Unión Europea, así como espacios en donde se está produciendo un proceso de sustitución entre estos colectivos?, y 3) la reactivación de los flujos de inmigración, ¿ha supuesto un cambio en el patrón territorial de localización de estos grupos dentro de la ciudad?

\section{La dinámica demográfica de la población extranjera en las áreas metropolitanas españolas}

Desde el comienzo de este siglo, las ciudades españolas han experimentado importantes cambios demográficos, y una gran parte de ellos está relacionado con los cambios residenciales de la población extranjera. Los análisis de distintos autores (Bayona-i-Carrasco, Gil-Alonso, Rubiales-Pérez y Pujadas-Rúbies, 2018; Bayona y Pujadas, 2020) han distinguido tres etapas en la evolución demográfica de las ciudades españolas en este periodo. La primera, que finaliza con la crisis económica de 2008 , se caracteriza por la aceleración 
migratoria, que conllevó la reversión de las pérdidas demográficas de los centros metropolitanos y la extensión y consolidación de las periferias, llevando a hablar de un cambio de modelo (Nel.lo, 2004). A partir de 2008 y hasta aproximadamente 2013, las consecuencias de la crisis económica y la reducción de la entrada de población en los espacios urbanos dio lugar a una fase de estancamiento demográfico, que afectó especialmente a la ciudad central y paralizó las tendencias de suburbanización. A partir de esta fecha, se empezaron a suavizar algunos de los efectos más graves de la crisis económica y la dinámica demográfica se caracterizó por un menor potencial de crecimiento y una reducción de las diferencias entre el centro y las periferias metropolitanas, debido no tanto al descenso de la intensidad de las migraciones residenciales, como a una mayor complejidad de los orígenes y destinos de los flujos. En los años más recientes, el centro sigue perdiendo población en la mayoría de las ciudades, mientras que las periferias muestran de nuevo valores positivos (Bayona et al., 2018).

Una parte sustancial de la estructura sociodemográfica urbana actual, por tanto, se deriva de las pautas de localización de la población extranjera llegada durante la fase de expansión económica, así como de los cambios residenciales que experimentaron en esa fase y durante la etapa de estancamiento demográfico posterior. El comportamiento de la población extranjera es especialmente relevante no sólo por representar un volumen importante de la población que llega a las ciudades desde principios de siglo, sino también porque ha mostrado un comportamiento diferencial tanto en su ubicación inicial en el entorno metropolitano como en cuanto a sus cambios residenciales posteriores a su arraigo. En relación con la localización inicial, la población recién llegada se concentra principalmente en las ciudades centrales de las áreas metropolitanas, buscando preferentemente los centros urbanos degradados, y revirtiendo así las tendencias a la pérdida de población de estos espacios centrales (Bayona, 2007; Bayona y López-Gay, 2011). Por otra parte, los inmigrantes han mostrado habitualmente tasas de movilidad residencial más altas que la población española, un fenómeno común a otros espacios urbanos (Rogers y Hennig, 1999; Bayona et al., 2018), por lo que, a medida que se incrementan los flujos, ha aumentado la difusión territorial de la inmigración en el espacio urbano, desplazándose en particular hacia la primera corona metropolitana.

Esta tasa de movilidad, sin embargo, experimenta cambios en función de la situación económica. Así, los cambios residenciales hacia las áreas suburbanas y barrios periféricos fueron especialmente intensos durante la etapa de crecimiento económico, en un proceso que se ha relacionado con su asimilación e integración social (Massey y Denton, 1985). Esta tendencia a una mayor movilidad se mantuvo además por más tiempo una vez llegada la crisis económica, a diferencia de la movilidad de la población española, que se empieza a reducir antes incluso de su comienzo. La llegada de la crisis supuso un descenso de las entradas de población extranjera, así como una importante reducción de su movilidad, en particular de la dirigida a la corona metropolitana (Bayona et al., 2018). Según estos autores, los movimientos metropolitanos de los extranjeros en 2012 habían decrecido un $19 \%$ en Madrid, un 25\% en Barcelona, y un $30 \%$ en Valencia. En consecuencia, se produjo un cambio en los patrones espaciales: por un lado, los centros de las ciudades comenzaron a perder menos población que en la etapa anterior; por otro, los municipios de la corona metropolitana recibieron menos población, y, en algunos casos, incluso presentaron tasas de migración neta negativas (López-Gay, 2014).

La fase de recuperación iniciada a partir de 2013 parece haber reactivado la tendencia a la suburbanización; o, al menos, así lo parecen indicar los análisis más recientes de la inmigración latinoamericana -que constituye una parte sustancial de la inmigración- referidos al periodo 2015-2017 en Madrid y Barcelona (Thiers-Quintana y Gil-Alonso, 2020). Esta reactivación de la suburbanización no se ha traducido, sin embargo, en un retroceso de la concentración en las ciudades centrales debido a la entrada de un mayor número de inmigrantes de este colectivo en las áreas metropolitanas estudiadas. Los autores relacionan esta tendencia con la teoría de la asimilación segmentada entendida en sentido amplio (Portes y Zhou, 1993), por la cual un mismo grupo de migrantes puede variar su comportamiento territorial en varios momentos, en función de la coyuntura económica, tendiendo a la concentración en los lugares centrales durante la recesión como una táctica defensiva frente a los impactos negativos de la crisis, e intensificando la tendencia a la suburbanización en las etapas de recuperación.

Debido probablemente a esta dinámica de dispersión y suburbanización, la llegada de este elevado volumen de población inmigrante no ha dado lugar a procesos de segregación especialmente acusados en las ciudades españolas. Distintas investigaciones realizadas en este sentido (Domínguez Mujica, Díaz Hernández y Parreño Castella- 
no, 2010; Sabater, Galeano y Domingo, 2013; Galeano y Bayona, 2018) concluyen que los niveles de concentración y segregación residencial en España son moderados, tanto si los comparamos con Estados Unidos como con otros países europeos, y han ido descendiendo a medida que aumentaban los flujos de entrada de extranjeros y la consiguiente redistribución territorial, aunque existen diferencias entre los distintos grupos nacionales. No obstante, la menor segregación residencial no significa que no existan problemas de integración, ya que, en España, como en otros países mediterráneos, los inmigrantes tienen, entre otros problemas, peores condiciones de vivienda, así como especiales dificultades de acceso a la misma (Malheiros, 2002). Por otra parte, la consistencia de estos resultados ha sido discutida debido a la falta de homogeneidad en la escala de análisis, la ausencia de datos demográficos comparables, y el escaso número de ciudades analizadas. De hecho, análisis más sistemáticos y a escala metropolitana han concluido que la complejidad y heterogeneidad de los patrones de segregación que muestran las ciudades mediterráneas hace necesario matizar y, en parte, corregir, la idea de la existencia de un bajo nivel de segregación en las ciudades meridionales (Benassi, Iglesias-Pascual, y Salvati, 2020).

En este sentido, distintos estudios (Musterd, Marcińczak, van Ham y Tammaru, 2017; Hochstenbach y Musterd, 2018; Lopez Gay, Andújar-Llosa y Salvati, 2020; Rubiales, 2020) han señalado una tendencia reciente al aumento de las desigualdades sociales y la diferenciación espacial en los entornos metropolita- nos que se relaciona con el proceso de globalización, y que afecta especialmente a la ciudad central debido a la expansión inmobiliaria, el posicionamiento de la ciudad en los flujos globales y, como una de sus consecuencias, al aumento de la demanda turística y los procesos de gentrificación. Los procesos de segregación actuales parecen pues más intensos en el caso de las clases altas (López Gay, et al., 2020; Rubiales, Bayona y Pujadas, 2012), lo que, en relación con los extranjeros, se asocia principalmente a los nacionales de Europa Occidental, cuyo perfil socioeconómico más elevado se combina con el descenso de su estructura de edad y su preferencia por barrios en proceso de gentrificación (Galeano y Bayona, 2018). Así, mientras que los espacios exclusivos se expanden rápidamente de la mano de una inmigración cada vez más cualificada, la población más vulnerable se ve desplazada y concentrada en espacios más periféricos, con peor acceso a todo tipo de servicios. Este proceso ha sido identificado tanto en distintas ciudades europeas (Musterd et al., 2017; Hochstenbach y Musterd, 2018) como en las principales áreas metropolitanas españolas (López-Gay et al., 2020; Rubiales, 2020; Salom y Pitarch, 2021), y justifica el renovado interés por el estudio de la diferenciación socioespacial de la población metropolitana.

\section{La población extranjera en la ciudad de Valencia}

Como otras ciudades españolas, el municipio de Valencia ha estado recibiendo importantes volúmenes de población extranjera desde principios de siglo, lo

FIGURA 1

EVOLUCIÓN DEL PORCENTAJE DE POBLACIÓN EXTRANJERA EN EL MUNICIPIO DE VALENCIA

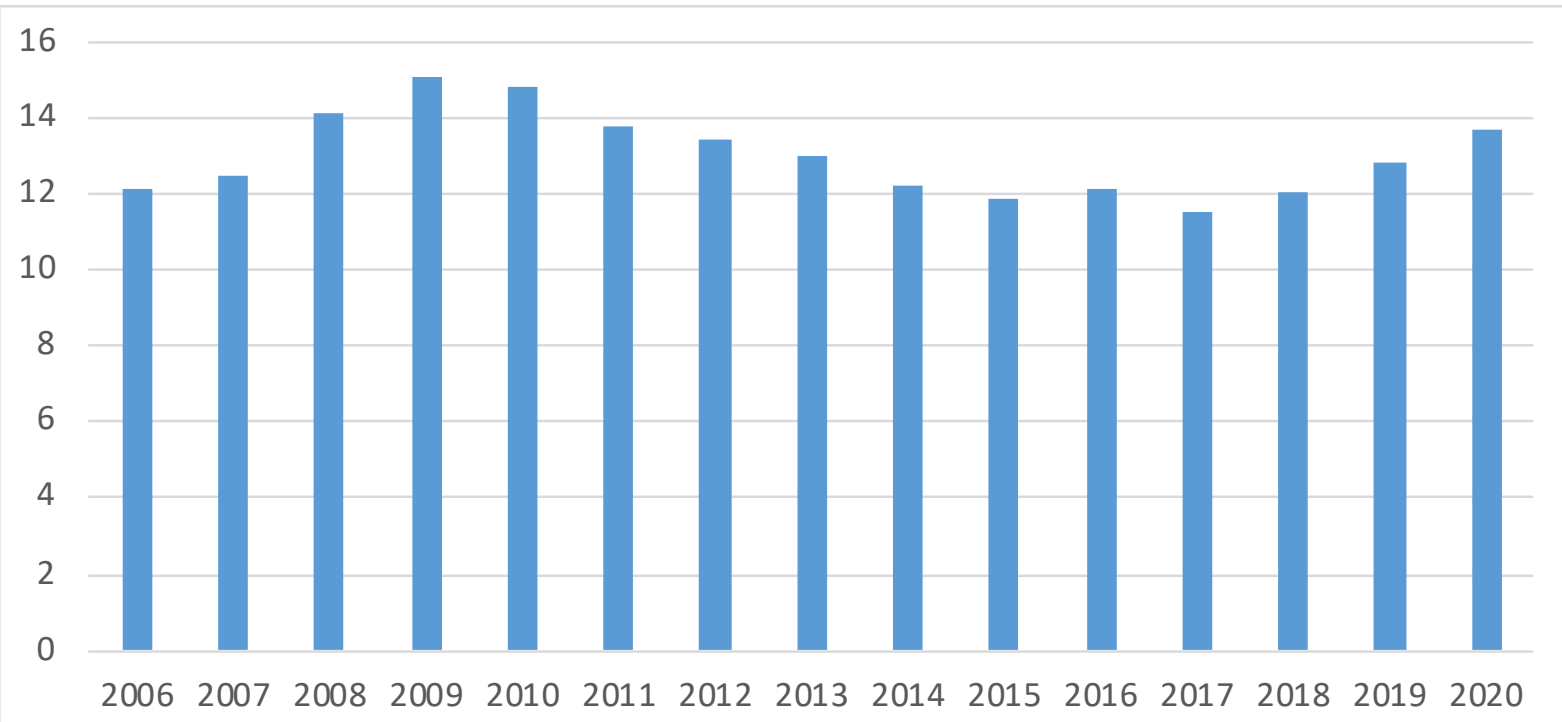

Fuente: INE, Padrón municipal de Población 
que ha hecho ascender progresivamente el porcentaje que supone la población extranjera sobre el total hasta un máximo del $15,06 \%$ en el año 2009. A partir de esta fecha, y como consecuencia de la reducción de las entradas y el aumento de las salidas, el porcentaje de población extranjera va disminuyendo, hasta estabilizarse en torno al $12 \%$ en el periodo 2015-2018, para remontar de nuevo en los dos últimos años registrados (Fig.1).

Los colectivos más importantes dentro de esta corriente migratoria son el latinoamericano, especialmente las personas de origen ecuatoriano, colombiano y boliviano, y el europeo, especialmente el rumano, y, en menor número, las personas de Bulgaria y Ucrania. Los inmigrantes de origen africano, principalmente de Marruecos, constituyen el grupo de procedencia por continente más antiguo. Nos encontramos, por tanto, ante una inmigración heterogénea, aunque el perfil mayoritario es el de una migración familiar (Torres, Pérez, Mut, Giner y Monsell, 2018). Como consecuencia de la crisis, parte de la inmigración latinoamericana ha retornado a los países de origen, aunque no siempre de modo definitivo, así como a otros países terceros, en su inmensa mayoría hacia países europeos con una mejor situación socioeconómica. En el caso de los europeos del Este, particularmente de la población rumana, es muy frecuente la migración circular entre Valencia y Rumanía, como estrategia para optimizar las ocasiones de trabajo en uno y otro espacio (Viruela y Torres, 2017). Sin embargo, a pesar de esta alta movilidad, la estadística de Variaciones Residenciales entre 2006 y 2016 indica que se han producido de manera global más Ilegadas que salidas, con sólo los años 2010, 2012 y 2013 mostrando saldos negativos.

En relación con su ubicación dentro de la ciudad, Torres et al. (2018) establecen tres periodos: En el primero, desde mediados de la década de los años 1980 hasta el final del siglo, en el que el número de extranjeros empadronados, fundamentalmente marroquíes y chinos, era muy reducido (en 1998 los extranjeros suponían el $1 \%$ de la población), los inmigrantes se instalan en tres tipos de espacios $\left.{ }^{2}: 1\right)$ los barrios más modestos del centro histórico, como El Pilar y El Mercat; 2) Barrios populares semi-centrales como Russafa y La Roqueta, cuya localización próxima a la estación de ferrocarril y la disponibilidad de bajos comerciales y vivienda barata favoreció el asentamiento de la población inmigrante, y 3) Barrios obreros periféricos, en donde existía una bolsa de vivienda barata, de vivienda VPO construida durante los años 1960 y 1970.
En el segundo período, desde el año 2000 hasta el 2010, el número de extranjeros aumenta exponencialmente, y su presencia se extiende a casi todos los barrios de la ciudad; el porcentaje de extranjeros en los barrios centrales o semi-centrales se estabiliza o desciende, y se incrementa en los barrios obreros y periféricos del norte, este y sur de la ciudad (Els Orriols, Cabanyal y Natzaret en los Poblats Marítims, Tres Forques y Fontsanta en el distrito de L'Olivereta; así como Cami Fondo, En Corts y El Calvari). Entre 2006 y 2010, a los anteriores se unen barrios vecinos como Torrefiel, La Creu del Grau, Mont-Olivet, Les Tendetes y Marxalenes. En esta etapa, como ha sucedido con otras grandes ciudades españolas, se produce también un proceso de desconcentración hacia la periferia metropolitana, aunque algo más tardío que el ocurrido en Madrid y Barcelona. La movilidad residencial de los extranjeros siguió creciendo hasta 2007, para luego descender, aunque en menor medida que en otras ciudades (Bayona et al., 2018).

Finalmente, en el tercer periodo, de 2010 a 2019, se consolida el patrón de distribución espacial del periodo anterior, de forma que, aunque la inmigración está presente en toda la ciudad, su presencia porcentual es más destacable en los distritos obreros periféricos del norte, este y oeste de la ciudad.

Estas pautas de localización se han traducido en unos niveles de segregación bajos, menores de los de otras ciudades españolas. Así, Domínguez et al. (2010) indican que en 2009 Valencia mostraba un índice de disimilitud de 0,23 , el más reducido de las ocho ciudades españolas estudiadas, y ofrecía también, junto con Palma y Murcia, una mejor situación en relación con la disponibilidad de superficie de vivienda por persona y tasas de hacinamiento a nivel de ciudad.

No obstante, y siguiendo la tendencia apuntada por el resto de ciudades europeas y españolas, la ciudad y su área metropolitana ha venido experimentado desde principios de siglo un aumento de la polarización geográfica y de los contrastes sociodemográficos internos (Salom y Fajardo, 2017). Tras la crisis económica de 2008, y fundamentalmente durante la segunda década del actual siglo, Valencia sufre las consecuencias de la recesión de forma más intensa que otras ciudades españolas. A partir de 2008, el área experimenta una evolución del mercado de trabajo más desfavorable que la media regional, lo que supone la inversión de una tendencia que se había mantenido durante décadas. Así, el índice de paro registrado sobre población potencialmente activa se triplica en 10 años, pasando del 5,5 al 15\% 
entre 2001 y 2012 (Salom y Pitarch, 2014). Los efectos negativos de la crisis han afectado especialmente a determinados municipios de la primera corona metropolitana, sobre todo de l'Horta Nord y Sud, aumentando así las desigualdades internas (Salom y Pitarch, 2014; Romero González, Salom-Carrasco, Albertos Puebla, Pitarch-Garrido y Melo Escrihuela, 2018). Por otra parte, en las fechas más recientes, se han consolidado procesos de reorganización interna relacionados con la turistificación, la gentrificación y los efectos sociales de determinados proyectos urbanísticos, lo que ha producido la concentración de grupos sociales similares en determinados barrios de la ciudad (Salom y Pitarch, 2021). La figura 2 muestra los contrastes internos en términos de renta que son el resultado de estas tendencias.

En las páginas siguientes analizamos la contribución de la población extranjera a estos procesos, e intentaremos averiguar si la movilidad residencial y migratoria de este grupo de población en estos últimos años caracterizados por una cierta recuperación económica ha contribuido o no a atenuar la concentración espacial prexistente, así como si han aparecido nuevas tendencias que pudieran contribuir a un cambio sustancial en la estructura socioeconómica espacial de la ciudad.

\section{FUENTES Y METODOLOGÍA}

\section{Fuentes}

Las principales fuentes estadísticas utilizadas en este artículo son el Padrón de Habitantes de 2016 y la información de microdatos de Variaciones Residenciales durante el periodo 2014-2017 proporcionada por la Oficina de Estadística del Ayuntamiento de Valencia. Estos microdatos contienen, entre otros aspectos, información sobre la nacionalidad en tres grandes grupos (españoles, nacionales de un país de la Unión Europea, y nacionales de un país de fuera de la Unión Europea) y el nivel educativo (Menor de 18 años; no sabe leer y escribir; Inferior a graduado escolar; Graduado escolar o equivalente; y Bachiller, FP segundo grado o títulos equivalentes o superiores) de la persona que cambia de residencia, ocasionando alta o baja en una sección censal concreta, así como el lugar de origen y/o destino de cada desplazamiento. A pesar de que la nacionalidad sólo está disponible para tres grandes grupos, la diferenciación por niveles educativos nos permite discriminar, en el caso de los extranjeros de la Unión Europea, entre la población procedente de países del Este (en este caso principal- mente rumanos), que habitualmente tiene menor nivel educativo, de la del resto de países europeos, en general con índices mayores de esta variable.

Ya que el objetivo es obtener una panorámica general de los cambios en el patrón territorial urbano de la población extranjera, en el análisis incluimos no sólo lo que específicamente puede denominarse movilidad residencial, es decir, los cambios de residencia intraurbanos, sino también las entradas y salidas de población hacia y desde el municipio, sea en relación con la corona metropolitana, sea respecto a otros destinos; hablamos pues aquí de movilidad residencial y migratoria (Palomares-Linares, Feria y Susino, 2017). Los datos proporcionados por el Ayuntamiento de Valencia incluyen las altas por otros motivos (en las que se desconoce el origen), y las bajas de las que no se conoce el destino o por omisión (emigraciones sin declarar, inscripciones que no han sido renovadas o dadas de baja), lo que nos permite incluir la entrada/ salida de población desde y hacia el extranjero en el saldo de la movilidad urbana ${ }^{3}$.

Las restricciones de la fuente nos obligan a utilizar la población de nacionalidad extranjera, lo que deja fuera del cómputo la población inmigrante que se ha nacionalizado española en los últimos años, que puede constituir una parte relativamente importante de la población de origen extranjero en algunos grupos nacionales; pero nos permite, por el contrario, incluir los hijos de inmigrantes nacidos en España que han conservado la nacionalidad original ${ }^{4}$. Por otra parte, esta fuente de información nos permite descender al detalle de la dinámica espacial de la movilidad residencial y migratoria a escala de sección censal, realizando una aproximación a las potenciales dinámicas sociales de segregación asociadas a través de la combinación de la variable nacionalidad con la variable nivel educativo, que a efectos del presente análisis hemos agrupado en dos categorías, según sea inferior o igual o superior a Bachiller, FP segundo grado y equivalentes. Debido a los cambios experimentados a lo largo de los años analizados y la necesidad de utilizar una división censal homogénea para el conjunto del periodo, se ha utilizado como base territorial la división censal de 2016 con la agregación de dos secciones censales del distrito 16 (las secciones 32 y 31, que se agregan respectivamente a la 29 y 30 del mismo distrito), tomando como referencia la información proporcionada por el Ayuntamiento de Valencia.

El análisis se ha realizado para el periodo comprendido entre el 1 de enero de 2014 y el 1 de enero de 2018, una etapa caracterizada por la reactivación 
FIGURA 2

RENTA MEDIA (MILES DE EUROS) EN 2015

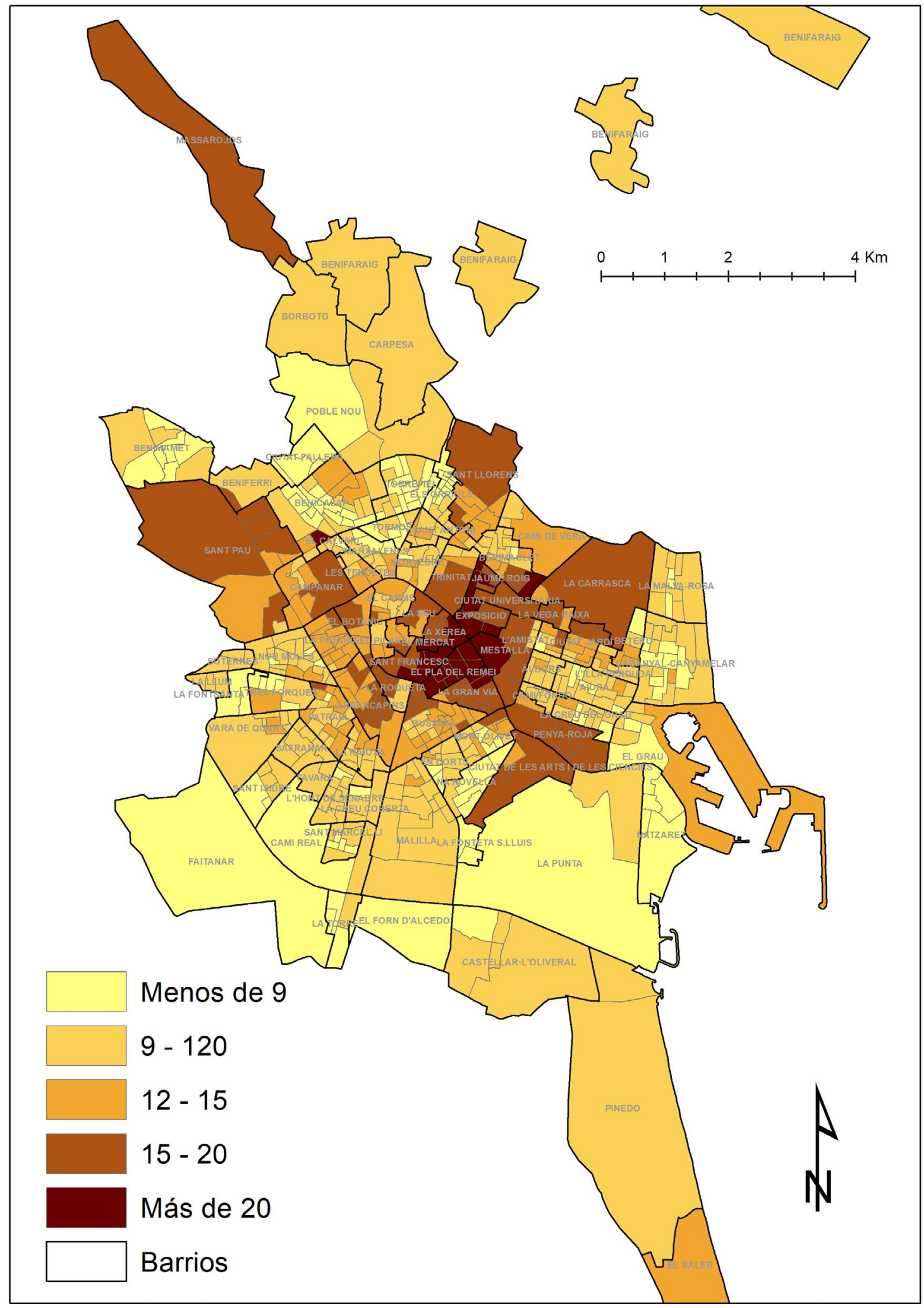

Fuente: INE, Atlas de distribución de renta de los hogares 
de la actividad económica y de los mercados inmobiliarios que se ha visto incentivada por un notable incremento en los precios de la vivienda y por la intensificación del desarrollo turístico de la ciudad (Méndez, 2019, p. 202). Además, el inicio del periodo coincide con el año a partir del cual la información de niveles educativos recogida en el padrón de habitantes tiene, según información proporcionada por el ayuntamiento, suficiente fiabilidad como para ser objeto de análisis. ${ }^{5}$

Por su parte, para la elaboración de la situación existente en 2016 se han utilizado los datos procedentes del Padrón de Población de 2016 proporcionados a nivel de sección censal por el Servicio de Estadística del Ayuntamiento de Valencia.

\section{Metodología}

El objetivo de este trabajo es identificar si la movilidad residencial de la población extranjera en estos años caracterizados por la recuperación económica está modificando o reafirmando el patrón territorial de localización preexistente de este colectivo, y si han aparecido nuevas tendencias que puedan contribuir a un cambio en la estructura socioeconómica espacial de la ciudad. Para dar respuesta a estos planteamientos, el análisis consta de tres pasos:

a) Identificación de los espacios de preferente localización de la población extranjera en 2016, diferenciando entre, por una parte, la población de países de fuera de la Unión Europea y, por otra parte, la población nacional de países de la Unión Europea. Se ha diferenciado, además, por nivel educativo, con el fin de distinguir, en la medida de lo posible, la población de países del este de Europa, en este caso principalmente de Rumanía, del resto de extranjeros europeos, procedentes mayoritariamente de Europa Occidental. El resultado nos muestra la imagen de la diferenciación espacial de partida existente en la ciudad en cuanto a localización de la población extranjera.

b) Análisis y cartografía de las tasas de cambio residencial y migratorio de los cuatro grupos de población extranjera considerados en el periodo analizado, para detectar los procesos de especialización/dispersión territorial que están operando en la ciudad en el periodo estudiado.

c) Comparación entre los resultados de los dos pasos anteriores, con el fin de identificar la existencia de un estancamiento o intensificación de la especialización residencial, así como potenciales cambios de tendencia significativos, y su localización en el territorio.

Para responder al primer punto, se ha aplicado un análisis de agrupamiento espacial (Grouping) a la distribución por grandes grupos nacionales de la población de 2016 a escala de secciones censales, con el fin de establecer una tipología de comportamiento que pueda apuntar a procesos sociales, económicos y residenciales diferenciados. Este análisis de agrupamiento considera, de forma similar a otros análisis cluster, la similitud de los casos a partir de un conjunto de variables, y los clasifica mediante un proceso de división iterativa; sin embargo, introduce una restricción espacial en la formación de los grupos, de forma que garantiza grupos compactos, formado por elementos contiguos o próximos (Lloyd, 2010; ESRI, 2021). En nuestro caso, se ha aplicado una restricción espacial de ocho elementos (8_Nearest_Neighbors), de acuerdo con la cual para adscribir una unidad territorial a un grupo determinado debe encontrarse en ese grupo al menos uno de los 8 vecinos más próximos.

Se han utilizado las siguientes variables: Porcentaje sobre la población total de los mayores de 25 años con nivel educativo de Bachiller, FP segundo grado o titulación equivalente o superior; porcentaje sobre la población total de extranjeros de países de fuera de la Unión Europea; porcentaje sobre la población total de extranjeros de países de la Unión Europea con nivel educativo inferior a Bachiller; y porcentaje sobre la población total de extranjeros de países de la Unión Europea con nivel educativo de Bachiller o más (ver tabla 1). La primera variable nos permite incorporar-de forma indirecta- información referida al nivel de renta de toda la población de la sección censal (incluidos los españoles), y nos indica el nivel socioeconómico de cada unidad territorial. Las otras tres variables se refieren al peso de cada uno de los tres grupos de extranjeros que utilizamos en el análisis sobre el total de la población de la unidad territorial. La diferenciación de la población de la UE por niveles educativos nos permite distinguir, de forma indirecta, entre la población extranjera vinculada a la inmigración de carácter económico (procedente fundamentalmente de Europa del Este) de los europeos de países con mayor nivel de renta, que tienen un comportamiento residencial diferenciado. En este análisis no se ha utilizado la diferenciación por niveles educativos de la población procedente de fuera de la Unión Europea ya que la incorporación de esta variable no mejoraba los resultados del análisis. 
En segundo lugar, los principales indicadores utilizados para determinar la tendencia a la "especialización" de cada sección censal en un determinado subgrupo de población han sido el saldo y tasa derivada de los cambios residenciales y migratorios de cada subgrupo de población. La localización geográfica de los saldos positivos de movilidad residencial nos permite elaborar un mapa de la tendencia a la "especialización" de cada una de las secciones censales en un subgrupo sociodemográfico determinado, lo que nos indica la posible existencia de dinámicas de concentración geográfica de determinados grupos nacionales. Por el contrario, la existencia de saldos negativos nos indica una tendencia a la salida de población, lo que puede apuntar a un proceso de desconcentración o relocalización de un grupo demográfico concreto.

La identificación de patrones espaciales y la detección de las áreas con cambio sociodemográfico significativo se ha basado en la aplicación de distintas técnicas fundamentadas en la autocorrelación espacial: cálculo del índice I de Moran Global y detección de clúster espaciales mediante Getis-Ord Hot Spot Analysis (Ord y Getis, 1995). La autocorrelación espacial permite medir la significación de la asociación entre valores próximos, sea a escala del conjunto del área de estudio, sea en función de la distancia que separa las observaciones, lo que permite detectar agrupaciones (cluster) locales de valores similares en magnitud.

El cálculo del índice global de autocorrelación espacial, o I Global de Moran se ha aplicado a las tasas migratorias en cada una de las categorías sociodemográficas estudiadas a distintas distancias, lo que ha permitido detectar simultáneamente si existe algún proceso de autocorrelación espacial en la distribución de los valores o si éstos son aleatorios, y, en el primer caso, a qué distancia este fenómeno actúa con un mayor nivel de significación. Por su parte, la aplicación del índice Getis-Ord Hot Spot Analysis o $\mathrm{GI}^{*}$ ha permitido localizar el agrupamiento de valores altos y bajos, e identificar así la localización de espacios concretos dentro de la ciudad que muestran un comportamiento específico en relación con los cambios residenciales. El valor del índice indica la existencia de un agrupamiento de valores altos (hot spots, valores positivos), o bajos (cold spots, valores negativos), midiéndose además el nivel de significación de dichos valores. La herramienta utilizada es el Optimized Hot Spot Analysis de ArcGis (Ord y Getis, 1995).
Finalmente, la constatación de la continuidad o no de los patrones espaciales se ha realizado mediante la superposición cartográfica de los cluster identificados en el paso anterior y la tipología resultante del análisis inicial. Esto nos ha permitido identificar el grado de coincidencia o no de ambos patrones espaciales, así como los espacios de dispersión residencial, en su caso, de los distintos grupos nacionales. Este análisis se ha realizado mediante sistemas de información geográfica (programa ArcGis 10.8 de Esri).

\section{RESULTADOS}

\section{Localización actual de la población extranjera}

La figura 3 muestra una aproximación a la estructura territorial socioeconómica de la ciudad en 2016 a través de la distribución espacial de la población extranjera en función de su nacionalidad (grandes grupos) y nivel de estudios. El análisis Grouping ha permitido identificar 15 grupos de secciones censales, cuyas características sociodemográficas se presentan en la tabla 1. En síntesis, esta tipología se resume en cinco grandes tipos:

A) Zonas con alto porcentaje de población con estudios superiores, y con escasa presencia de población extranjera, aunque en algunos casos se detecta cierta presencia de nacionales de la UE de alto nivel educativo: Barrios de Sant Pau; área de Gran ViaExposició-Mestalla-Ciutat de les Arts i les Ciencies, y Arrancapins-La Roqueta (zonas 1, 8, y 10)

B) Zonas con alto porcentaje de población con estudios superiores, y con elevada presencia de población extranjera de todas las nacionalidades, pero especialmente procedente de países de la UE: Barrios de Russafa, Ciutat Vella y El Botánic (zonas 2, 3 y 6)

C) Zonas con predominio de la población con bajo nivel educativo y con altos porcentajes de población de la UE con bajo nivel educativo: Cabanyal, NatzaretLa Punta, y otros barrios de los Poblados Marítimos (zonas $4,5,7$ y 11 )

D) Zonas con predominio de población con bajo nivel educativo y alto porcentaje de extranjeros de fuera de la UE: Avenida del Puerto-El Grau, TorrefielEls Orriols, y Nou Moles-Soternes-La Llum (zonas 9, 12 y 13$)$

E) Zonas que presentan valores en torno a la media en todas las variables analizadas: barrios de Marxalenes-Tendetes-Benimaclet-Camí de Vera, y zona Norte y Suroeste de la ciudad (zonas 14 y 15). 
FIGURA 3

TIPOLOGÍA DE SECCIONES CENSALES SEGÚN EL NIVEL EDUCATIVO Y NACIONALIDAD DE LA POBLACIÓN RESIDENTE EN 2016

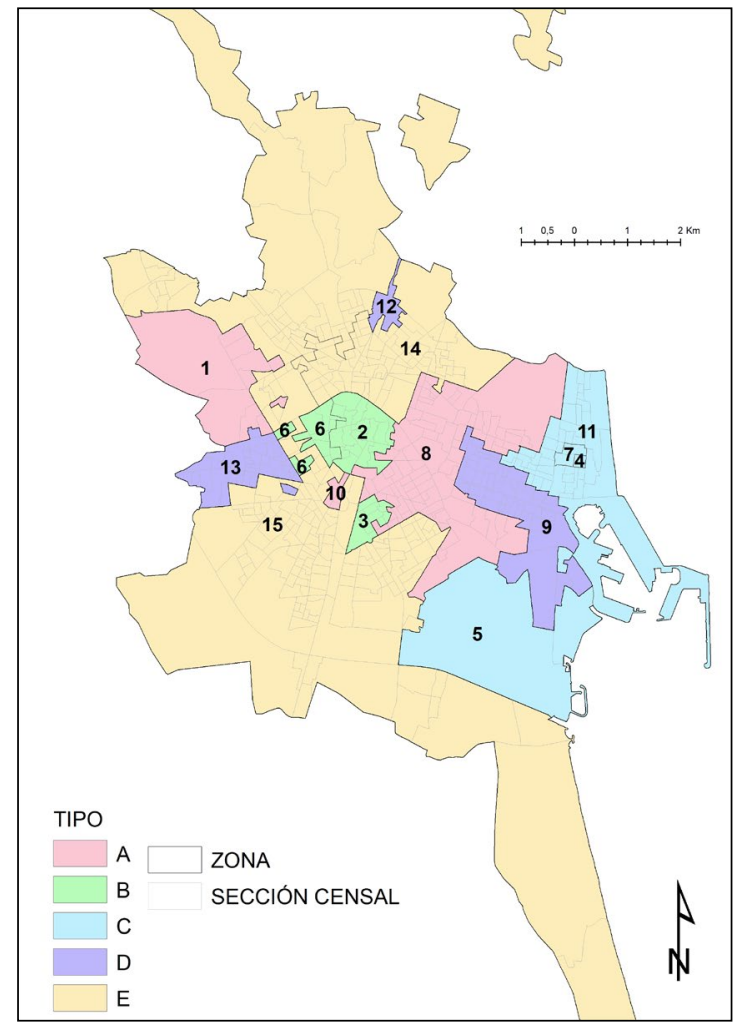

Los números y letras se corresponden con la tipología de la tabla 1.

Fuente: Padrón de Población. Ayuntamiento de Valencia
Estos resultados muestran el predominio superficial de las zonas caracterizadas por elevada presencia de población educada, así como los distintos niveles de segregación de la población extranjera en función de su origen geográfico. Así, llama la atención la dispersión territorial de la población extranjera de países de fuera de la UE, presente en la mayor parte del territorio urbano, aunque con mayor representación en los barrios periféricos, frente a la concentración geográfica de la población procedente de países de la Unión Europea. El elevado grado de segregación de este colectivo atañe tanto a la población con mayor nivel educativo, ubicada preferentemente en los barrios centrales con tendencia a la gentrificación (Ciutat Vella, Russafa y el Botánic), como a la población con menor nivel educativo (fundamentalmente de nacionalidad rumana), que se ubica preferentemente en siete secciones censales del barrio de El Cabanyal, en una de los cuales supone el $29 \%$ del total. Esta última situación, relacionada con los efectos negativos de la paralización del proyecto urbanístico de prolongación de la avenida de Blasco lbáñez (Ilisei y Salom, 2018), constituye el mayor problema relacionado con la segregación que tiene actualmente la ciudad (Ajuntament de Valencia, 2015).

Sobre esta estructura territorial están actuando nuevos procesos que pueden dar lugar a algunos cambios territoriales, a los que hacemos referencia a continuación.

TABLA 1

VALORES MEDIOS DE LOS INDICADORES UTILIZADOS PARA LA CLASIFICACIÓN

\begin{tabular}{|c|c|c|c|c|c|c|c|c|}
\hline \multirow[b]{2}{*}{ TIPO } & \multirow[b]{2}{*}{ GRUPO } & \multicolumn{2}{|c|}{$\begin{array}{l}\text { SECCIONES } \\
\text { CENSALES }\end{array}$} & \multirow[b]{2}{*}{ BARRIOS } & \multicolumn{4}{|c|}{ PORCENTAJE SOBRE LA POBLACIÓN TOTAL DE LA SECCIÓN } \\
\hline & & № & $\%$ & & $\begin{array}{l}\text { Población } \\
\text { con nivel } \\
\text { educativo } \\
\text { superior (\%) }\end{array}$ & $\begin{array}{c}\text { Nacionales } \\
\text { de países } \\
\text { fuera de la } \\
\text { UE (\%) }\end{array}$ & $\begin{array}{l}\text { Nacionales } \\
\text { de la UE con } \\
\text { menor nivel } \\
\text { educativo }\end{array}$ & $\begin{array}{l}\text { Nacionales de } \\
\text { la UE con nivel } \\
\text { educativo } \\
\text { superior }\end{array}$ \\
\hline \multirow{3}{*}{ A } & 1 & 10 & 1,68 & Sant Pau & 70,91 & 4,66 & 1,28 & 2,46 \\
\hline & 8 & 88 & 14,81 & Gran Vía-Exposició-Mestalla-Ciutat de les Arts i les Ciencies & 75,57 & 4,65 & 1,1 & 2,73 \\
\hline & 10 & 5 & 0,84 & Arrancapins-La Roqueta & 72,85 & 3,23 & 0,74 & 1,49 \\
\hline \multirow{3}{*}{ B } & 2 & 19 & 3,2 & Ciutat Vella & 69,59 & 7,08 & 2,55 & 6,39 \\
\hline & 3 & 10 & 1,68 & Russafa & 60,66 & 9,88 & 2,32 & 5,06 \\
\hline & 6 & 15 & 2,53 & El Botanic & 63,43 & 6,92 & 2,42 & 3,42 \\
\hline \multirow{4}{*}{ C } & 4 & 1 & 0,17 & Zona 0 Cabanyal (centro) & 26,44 & 4,15 & 28,85 & 1,84 \\
\hline & 5 & 3 & 0,51 & Natzaret-La Punta & 17,6 & 7,27 & 10,68 & 1,24 \\
\hline & 7 & 3 & 0,51 & Zona 0 Cabanyal (periferia) & 25,98 & 5,42 & 9,9 & 2,02 \\
\hline & 11 & 42 & 7,07 & Poblados Marítimos & 36,31 & 5,45 & 2,68 & 2,02 \\
\hline \multirow{3}{*}{ D } & 9 & 51 & 8,59 & Avenida del Puerto-El Grau & 38,45 & 11,1 & 3,07 & 2,03 \\
\hline & 12 & 12 & 2,02 & Torrefiel-Els Orriols & 21,76 & 19,6 & 3,4 & 1,33 \\
\hline & 13 & 36 & 6,06 & Nou Moles-Soternes-La Llum & 38,04 & 12,28 & 2,4 & 1,3 \\
\hline \multirow{2}{*}{$\mathrm{E}$} & 14 & 80 & 13,47 & Marxalenes-Tendetes-Benimaclet-Camí de Vera & 45,82 & 9,96 & 2,18 & 2,14 \\
\hline & 15 & 219 & 36,87 & Norte y Suroeste & 40,68 & 7,94 & 1,89 & 1,31 \\
\hline TOTAL & & 594 & 100 & TOTAL & 47,9 & 8,18 & 2,18 & 2,05 \\
\hline
\end{tabular}

Fuente: Ayuntamiento de Valencia, Padrón de Población, 2016 


\section{La movilidad residencial y migratoria de la población extranjera}

El periodo analizado se caracteriza por un crecimiento demográfico suave, debido a un saldo vegetativo crecientemente negativo, y un saldo migratorio crecientemente positivo. Tal y como puede verse en la tabla 2 , este saldo migratorio positivo se debe sobre todo a la entrada de población extranjera, mientras que el saldo global durante este periodo de la movilidad de la población española es negativo. Por otra parte, la distribución por nacionalidad de los movimientos residenciales internos (entre secciones y/o en el interior de la sección) nos muestra una gran movilidad residencial; se producen más de 40.000 cambios de residencia anuales en el periodo estudiado, lo que supone una tasa de movilidad de la población del 20,3\%; es decir, uno de cada cinco habitantes de la ciudad ha realizado un cambio de residencia a lo largo de estos cuatro años. La mayor parte de estos cambios corresponden a los realizados por

\section{FIGURA 4}

TASAS MIGRATORIAS (KRIGING) POR SECCIÓN CENSAL DE LOS NACIONALES DE PAÍSES DE FUERA DE LA UNIÓN EUROPEA CON ALTO NIVEL EDUCATIVO 2014-2017

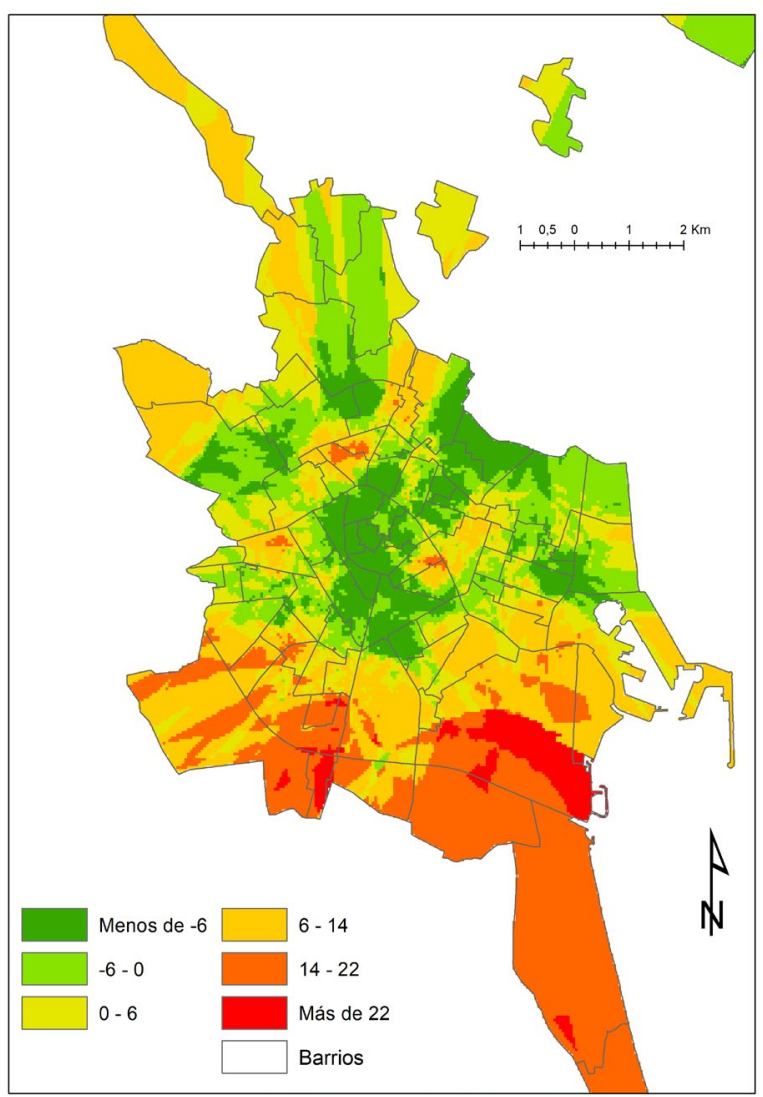

Fuente: Ayuntamiento de Valencia la población española (26.543 desplazamientos anuales), aunque la tasa de movilidad, es decir, el número de desplazamientos por persona, es mayor entre la población extranjera, en particular la procedente de países no europeos; una característica común a otras ciudades, como ya se ha comentado.

TABLA 2

MOVIMIENTOS DE POBLACIÓN EN EL MUNICIPIO DE VALENCIA EN EL PERIODO 2014-2017.

\begin{tabular}{|l|l|c|c|c|}
\hline \multicolumn{2}{|c|}{ TIPO DE MOVIMIENTO } & ESPAÑOLES & EXTRANJEROS & TOTAL \\
\hline \multirow{3}{*}{$\begin{array}{l}\text { MOVIMIENTOS } \\
\text { MIGRATORIOS }\end{array}$} & ALTAS & 80.919 & 70.808 & 151.727 \\
\cline { 2 - 5 } & BAJAS & 81.925 & 54.589 & 136.514 \\
\cline { 2 - 5 } & SALDO & -1.006 & 16.219 & 15.213 \\
\cline { 2 - 5 } & TASA (\%) & $-0,14$ & 0,17 & 1,92 \\
\hline \multirow{2}{*}{$\begin{array}{l}\text { CAMBIOS DE } \\
\text { DOMICILIO }\end{array}$} & NÚMERO & 106.175 & 54.286 & 160.461 \\
\cline { 2 - 5 } & TASA (\%) & 15,29 & 55,92 & 20,27 \\
\hline
\end{tabular}

Fuente: Ayuntamiento de Valencia. Microdatos de variaciones residenciales, varios años.

FIGURA 5

TASAS MIGRATORIAS (KRIGING) POR SECCIÓN CENSAL DE LOS NACIONALES DE PAÍSES DE FUERA DE LA UNIÓN EUROPEA CON BAJO NIVEL EDUCATIVO 2014-2017

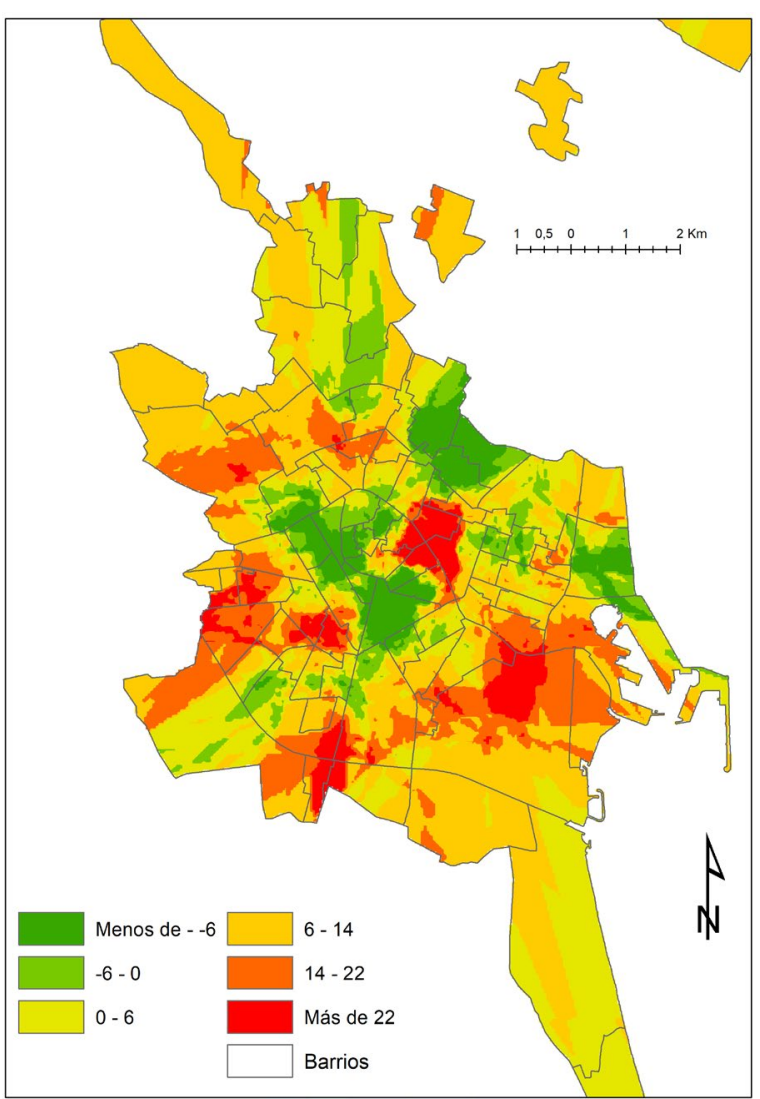

Fuente: Ayuntamiento de Valencia 
TABLA 3

MOVIMIENTOS DE LA POBLACIÓN SEGÚN LA NACIONALIDAD (ESPAÑOLA/RESTO DE LA UNIÓN EUROPEA/FUERA DE LA UNIÓN EUROPEA) Y EL NIVEL EDUCATIVO (MENOR DE 18 AÑOS/INFERIOR A BACHILLER/BACHILLER Y MÁS) EN EL PERIODO 2014-2017

\begin{tabular}{|c|c|c|c|c|c|c|}
\hline \multirow{2}{*}{ NACIONALIDAD/NIVEL EDUCATIVO } & \multicolumn{4}{|c|}{ MOVIMIENTOS MIGRATORIOS } & \multicolumn{2}{|c|}{ CAMBIOS RESIDENCIALES } \\
\hline & ALTAS & BAJAS & SALDO & TASA (\%) & NÚMERO & TASA (\%) \\
\hline UNIÓN EUROPEA (28) & 19.163 & 14.911 & 4.252 & 12,94 & 10.531 & 32,04 \\
\hline Menor de 18 años & 2.568 & 1.949 & 619 & 8,04 & 1.838 & 23,87 \\
\hline Inferior a Bachiller & 6.389 & 7.467 & -1.078 & $-8,31$ & 4.387 & 33,82 \\
\hline Bachiller y más & 10.206 & 5.495 & 4.711 & 38,62 & 4.306 & 35,3 \\
\hline FUERA DE LA UE & 51.645 & 39.678 & 11.967 & 18,64 & 43.755 & 68,14 \\
\hline Menor de 18 años & 8.234 & 4.868 & 3.366 & 20,74 & 7.055 & 43,47 \\
\hline Inferior a Bachiller & 25.152 & 24.062 & 1.090 & 3,24 & 26.393 & 78,53 \\
\hline Bachiller y más & 18.259 & 10.748 & 7.511 & 52,26 & 10.307 & 71,71 \\
\hline ESPAÑOLES & 80.919 & 81.925 & -1.006 & $-0,14$ & 106.175 & 15,29 \\
\hline Menor de 18 años & 14.831 & 15.191 & -360 & $-0,22$ & 24.702 & 15,39 \\
\hline Inferior a Bachiller & 28.602 & 32.639 & -4.037 & $-1,49$ & 41.853 & 15,49 \\
\hline Bachiller y más & 37.486 & 34.095 & 3.391 & 1,29 & 39.620 & 15,02 \\
\hline TOTAL & 151.727 & 136.514 & 15.213 & 1,92 & 160.461 & 20,27 \\
\hline Menor de 18 años & 25.633 & 22.008 & 3.625 & 1,97 & 33.595 & 18,22 \\
\hline Inferior a Bachiller & 60.143 & 64.168 & -4.025 & $-1,27$ & 72.633 & 22,92 \\
\hline Bachiller y más & 65.951 & 50.338 & 15.613 & 5,38 & 54.233 & 18,68 \\
\hline
\end{tabular}

Fuente: Microdatos de Variaciones Residenciales. Servicio de Estadística del Ayuntamiento de Valencia

FIGURA 6

TASAS MIGRATORIAS (KRIGING) POR SECCIÓN CENSAL DE LOS NACIONALES DE PAÍSES DE LA UNIÓN EUROPEA CON ALTO NIVEL EDUCATIVO (2014-2017)

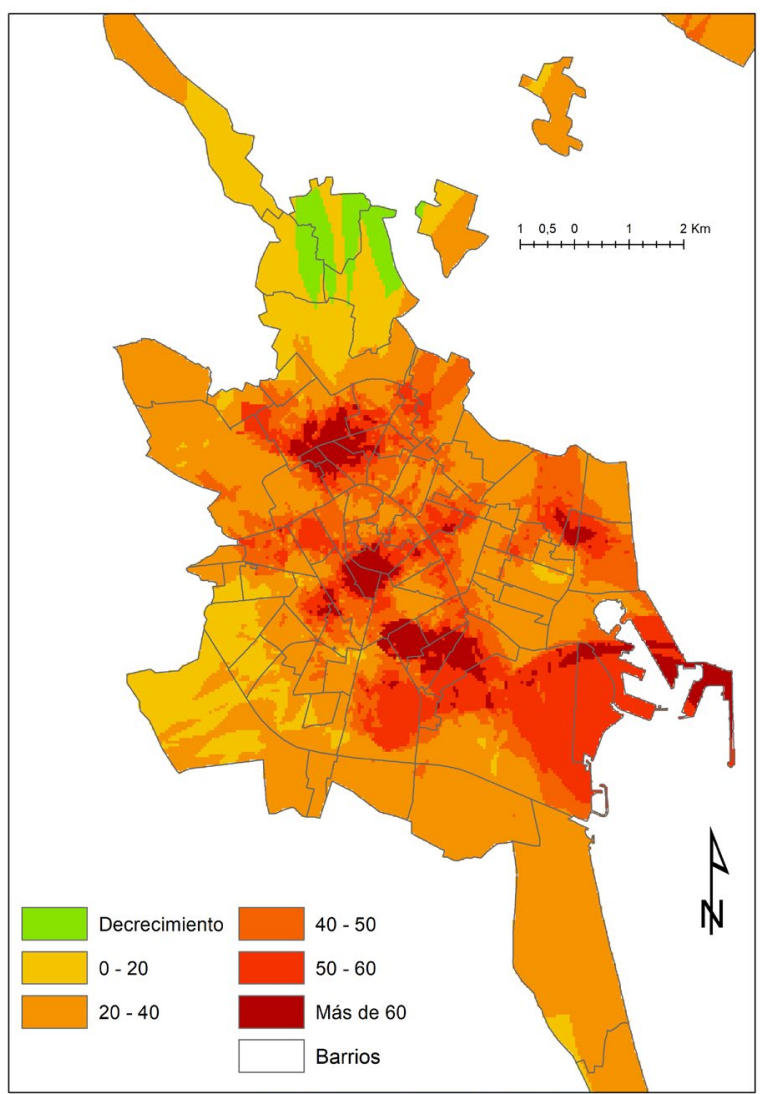

FIGURA 7

TASAS MIGRATORIAS (KRIGING) POR SECCIÓN CENSAL DE LOS NACIONALES DE PAÍSES DE LA UNIÓN EUROPEA CON BAJO NIVEL EDUCATIVO (2014-2017)

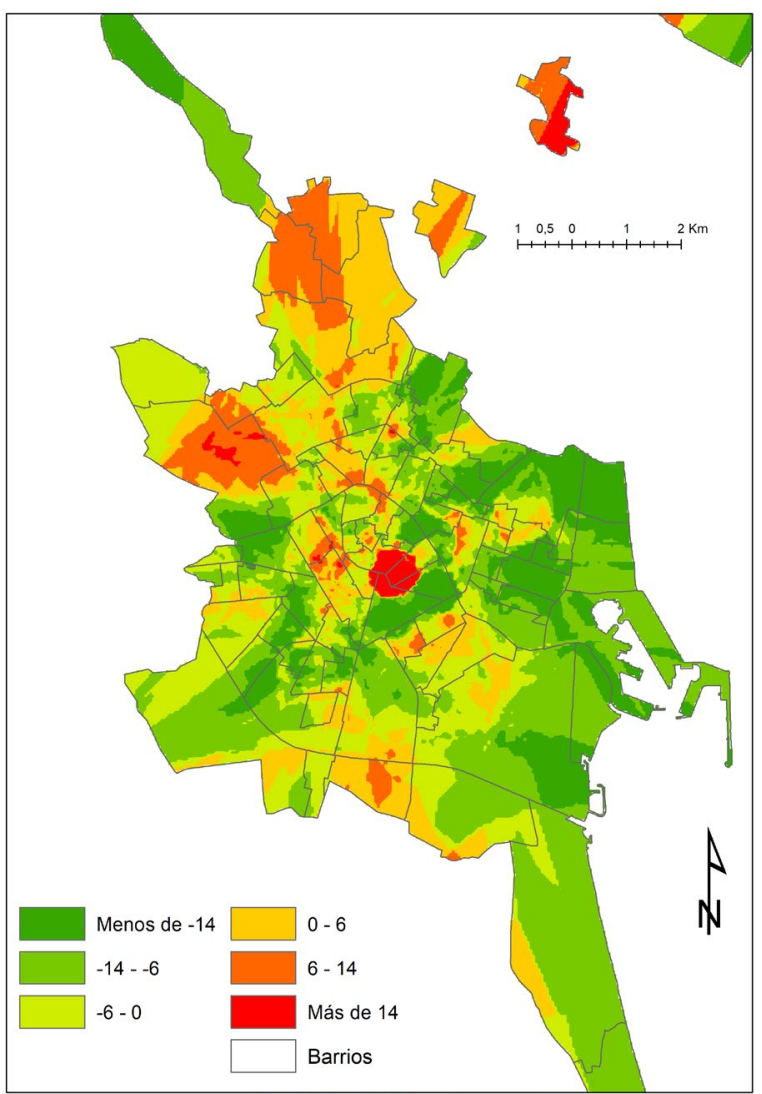


Centrándonos en la movilidad de la población extranjera (Tabla 3), son los nacionales de países de fuera de la UE los que están contribuyendo en mayor medida al crecimiento migratorio en la ciudad, caracterizándose esta inmigración por su carácter familiar, como lo demuestra el destacable número de entradas de personas con menos de 18 años. En conjunto, aunque por el número de entradas destaca la población de fuera de la UE con menor nivel educativo, ésta es también la que protagoniza el mayor número de salidas, por lo que el saldo supone una recualificación neta de la población extranjera municipal.

El patrón territorial de estos movimientos residenciales supone la conformación de zonas atractivas para determinados grupos nacionales, que muestran tasas migratorias netas positivas, y zonas de expulsión, con salida neta de población. Las figuras 4 a 7 nos muestran su localización geográfica mediante una representación suavizada que permite observar las tendencias espaciales ${ }^{6}$. Como puede observarse, los patrones espaciales son marcadamente contrastados, tanto entre nacionalidades (dentro y fuera de la Unión Europea) como entre niveles educativos.

Así, los nacionales de países de fuera de la Unión Europea tienden a mostrar una localización periférica, con tasas negativas en el centro urbano y positivas en los barrios periféricos, mientras que la población de la Unión Europea, sobre todo la de nivel educativo más elevado, tiende a concentrarse en los espacios centrales de la ciudad, agrupándose en torno a varios puntos concretos.

Para contrastar si estas tendencias espaciales que es posible constatar a simple vista son estadísticamente significativas, se ha aplicado un análisis de autocorrelación espacial de Moran a las tasas migratorias totales de los cuatro grupos de extranjeros considerados. Este tipo de análisis nos permite identificar si existe un patrón significativo de concentración en torno a determinadas áreas urbanas, identificando los espacios de concentración de valores negativos (zonas de expulsión) y positivos (zonas de atracción) de determinados grupos socioeconómicos. Este análisis no ha dado resultados significativos para los movimientos de población de los nacionales de la Unión Europea con bajo nivel educativo, lo que significa que no es posible identificar patrones de agrupamiento de valores positivos o negativos estadísticamente significativos a escala de sección censal para este grupo sociodemográfico. ${ }^{7}$ Por el contrario, el resultado ha sido positivo en el caso de los otros tres colectivos, por lo que podemos inferir que en estos casos sí que existe una tendencia espacial estadísticamente significativa. En consecuencia, se ha aplicado el análisis Getis-Ord a la movilidad de es- tos tres últimos grupos, con el fin de detectar los clusters territoriales que suponen zonas atractivas (en rojo) o repulsivas (en azul) para estos grupos nacionales durante el periodo estudiado. Los resultados se representan en las figuras 8 a 10.

La comparación de estos clusters con la tipología del apartado anterior nos permite detectar los casos en que la movilidad residencial está reforzando el modelo territorial anterior, así como las áreas urbanas en donde se están produciendo cambios. Comentaremos en primer lugar las áreas en que el saldo migratorio está provocando un incremento de la presencia de un grupo de extranjeros determinado.

En el caso de la población de la Unión Europea con alto nivel educativo, existen tres tipos de áreas que constituyen espacios atractivos:

a) Zonas de concentración tradicional de este tipo de población (tipo $B$ ), que siguen recibiendo volúmenes crecientes de población de este perfil; se trata de secciones ubicadas en los barrios de Ciutat Vella, Russafa y Gran Vía, que constituyen un $13 \%$ de las secciones identificadas como de concentración significativa de este grupo de población.

b) Secciones contiguas a las anteriores, en las que predomina una población con alto nivel educativo pero que tienen un todavía escaso peso de población extranjera (tipo A) o cuyas características se encuentran en torno a la media de la ciudad (tipo E). Esta zona de ampliación constituye un 20,6\% del total.

c) Nuevas áreas atractivas, ubicadas en zonas no especializadas (tipo E) o con predominio de población con bajo nivel educativo (tipos C-D) en las que la entrada de este perfil de población supone una recualificación ligada en algunos casos a promociones inmobiliarias o proyectos urbanísticos de renovación urbana. Encontramos tres áreas de este tipo: Una zona al norte del río que constituyó uno de los espacios de recepción inicial de la población inmigrante de bajo nivel educativo y que actualmente se encuentra en vías de transformación (barrios de Marxalenes, les Tendetes, Benicalap, Torrefiel, Tormos y El Calvari); una segunda zona en los barrios de Na Rovella, En Corts, y Monteolivet, próxima a barrios ya gentrificados como Russafa y Ciutat de les Arts i les Ciencies; y, finalmente, el área de Nazaret-La Punta-Grao, un espacio que se caracteriza por la alta presencia de población inmigrante con bajo nivel educativo, pero en donde se han desarrollado nuevos proyectos de urbanización. En su conjunto, estas nuevas zonas de crecimiento de la población europea suponen un $72,6 \%$ de la superficie y un $66,6 \%$ 
FIGURA 8

CLUSTER SIGNIFICATIVOS DE LAS TASAS MIGRATORIAS TOTALES DE LOS NACIONALES DE PAÍSES DE LA UNIÓN EUROPEA CON ALTO NIVEL EDUCATIVO

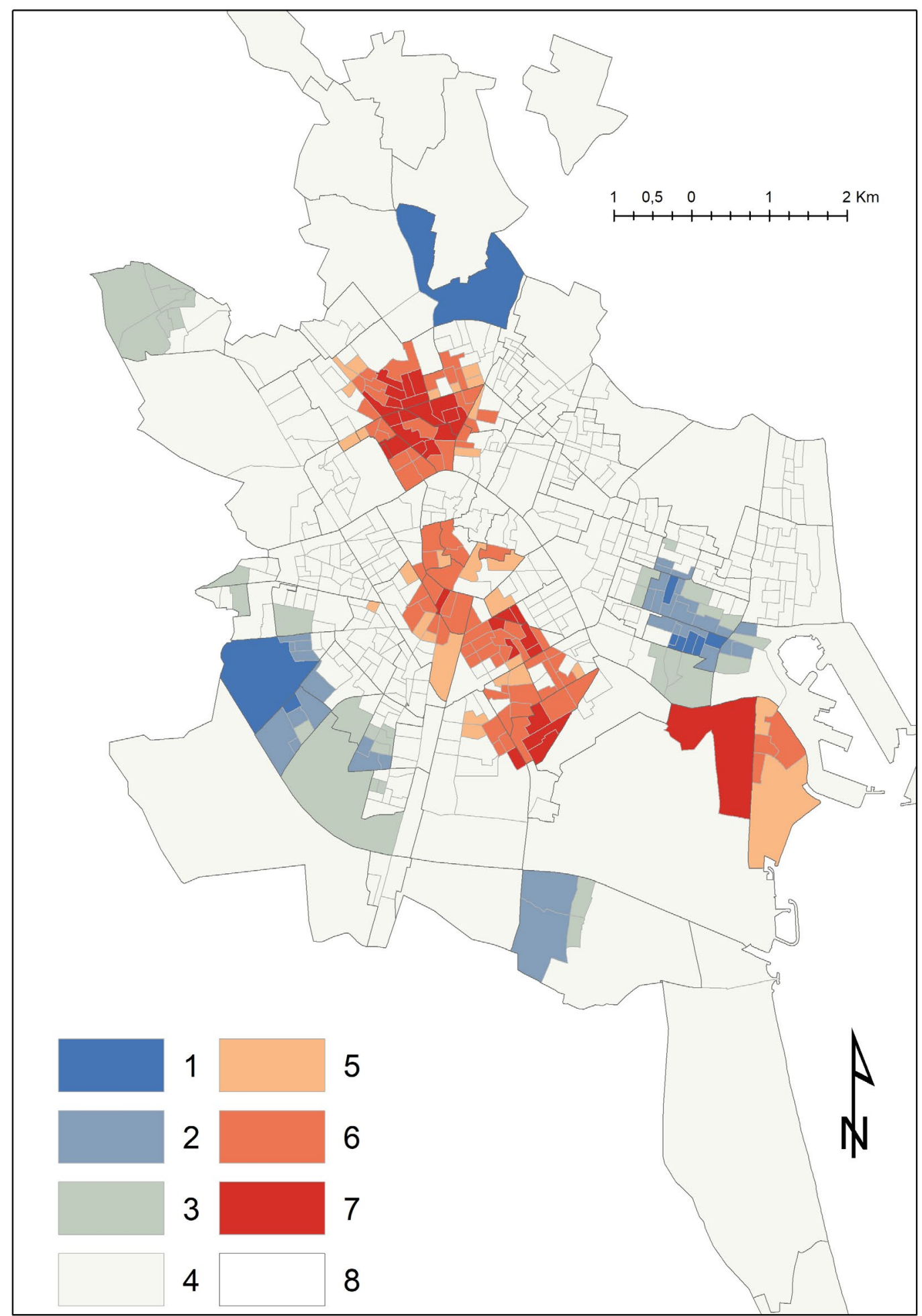

1.- Agrupaciones de valores negativos (Cold Spot) significativas al 99\%. 2.- Agrupaciones de valores negativos (Cold Spot) significativas al 95\%. 3.- Agrupaciones de valores negativos (Cold Spot) significativas al 90\%. 4.- No significativo. 5.- Agrupaciones de valores positivos (Hot Spot) significativas al 90\%. 6.- Agrupaciones de valores positivos (Hot Spot) significativas al 95\%. 7.- Agrupaciones de valores positivos (Hot Spot) significativas al 99\%. 8.- Límite de barrio. 
FIGURA 9

CLUSTER SIGNIFICATIVOS DE LAS TASAS MIGRATORIAS TOTALES DE LOS NACIONALES DE PAÍSES DE FUERA DE LA UNIÓN EUROPEA CON BAJO NIVEL EDUCATIVO

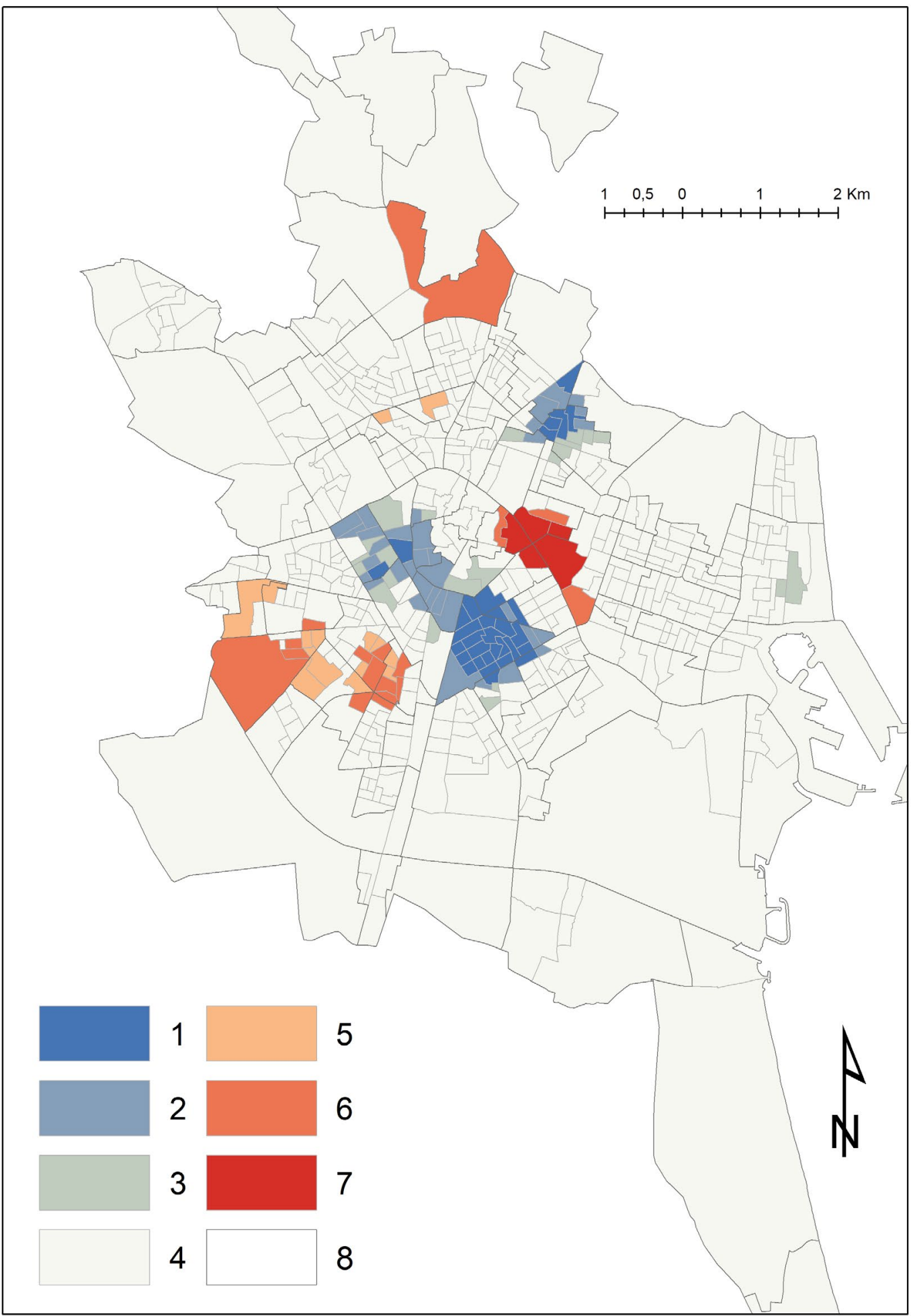

1.- Agrupaciones de valores negativos (Cold Spot) significativas al 99\%. 2.- Agrupaciones de valores negativos (Cold Spot) significativas al 95\%. 3.- Agrupaciones de valores negativos (Cold Spot) significativas al 90\%. 4.- No significativo. 5.- Agrupaciones de valores positivos (Hot Spot) significativas al 90\%. 6.- Agrupaciones de valores positivos (Hot Spot) significativas al 95\%. 7.- Agrupaciones de valores positivos (Hot Spot) significativas al 99\%. 8.- Límite de barrio. 
FIGURA 10

CLUSTER SIGNIFICATIVOS DE LAS TASAS MIGRATORIAS TOTALES DE LOS NACIONALES DE PAÍSES DE FUERA DE LA UNIÓN EUROPEA CON ALTO NIVEL EDUCATIVO

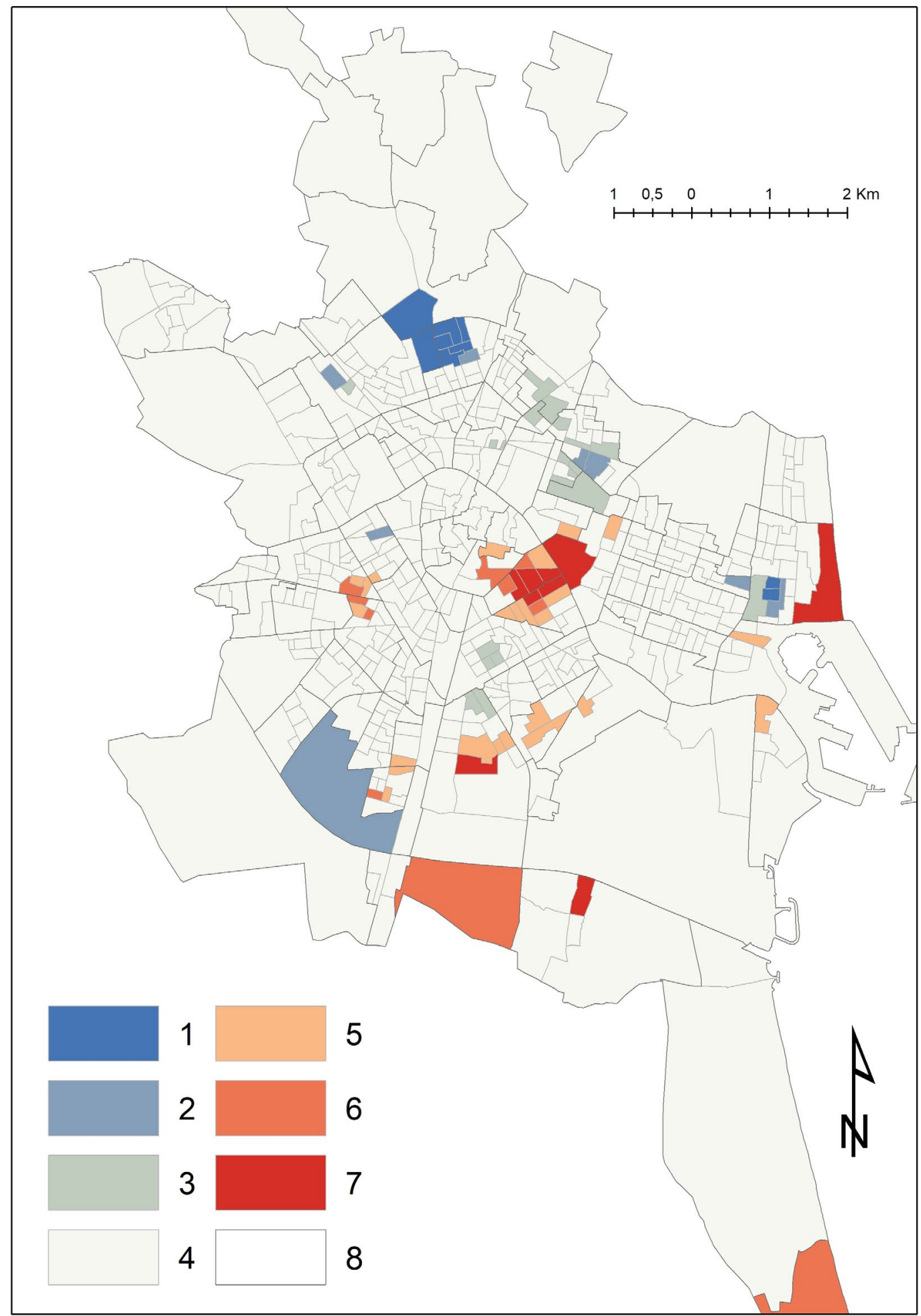

1.- Agrupaciones de valores negativos (Cold Spot) significativas al 99\%. 2.- Agrupaciones de valores negativos (Cold Spot) significativas al 95\%. 3.- Agrupaciones de valores negativos (Cold Spot) significativas al 90\%. 4.- No significativo. 5.- Agrupaciones de valores positivos (Hot Spot) significativas al 90\%. 6.- Agrupaciones de valores positivos (Hot Spot) significativas al 95\%. 7.- Agrupaciones de valores positivos (Hot Spot) significativas al 99\%. 8.- Límite de barrio. 
de las secciones con dinámicas de concentración significativa de la población de este perfil.

Por su parte, la población extranjera de países de fuera de la UE con nivel educativo alto muestra un patrón de concentración más polarizado y menos disperso que en el caso anterior. Los principales espacios atractivos son de dos tipos:

a) Por un lado, secciones censales ubicadas en los barrios de Pla del Remei y Gran Vía, zonas tipo A, es decir, con predominio de población educada, aunque todavía con escasa presencia de población extranjera. Este tipo de áreas constituye la mitad de las secciones censales atractivas para este perfil de población.

b) Por otra parte, varios clusters positivos que se ubican en su mayoría en la periferia de la ciudad, en áreas que se caracterizan hasta el momento por una escasa especialización en población extranjera de alto nivel educativo, y que constituyen nuevas zonas de expansión para este grupo sociodemográfico. Entre ellos, encontramos secciones concretas del frente marítimo, entre las que destacan los casos del barrio de El Saler, en la periferia meridional, y el frente marítimo del barrio del Cabanyal-Canyamelar (zona 11), así como clusters ubicados en la periferia metropolitana, en áreas con presencia de población de bajo nivel educativo, como Nou Moles-Patraix y Malilla-Na Rovella; Forn d'Alcedo, o San Marcelino.

Finalmente, la población extranjera de esta misma procedencia geográfica con bajo nivel educativo muestra, con escasas excepciones, una tendencia a la concentración en las áreas periféricas ya especializadas en este tipo de población (La Fontsanta-Tres Forques), así como en zonas próximas en donde la población extranjera es menos importante (clusters de Vara de Quart-Safranar y Patraix-La Raiosa, en el suroeste de la ciudad, y secciones de Poble Nou, Tormos y Marxalenes). La excepción a esta tendencia al refuerzo del patrón espacial existente es el clúster de Exposició-Mestalla, ubicado en barrios de tipo A, es decir, una zona con escasa presencia de población inmigrante, que es además atractiva también para la población extranjera de mayor nivel educativo.

Por otro lado, las zonas que mayoritariamente expulsan población extranjera están relacionadas con la presencia, mayoritaria o creciente, de población de un distinto perfil socioeconómico. Así, en el caso de la población de la UE, los principales clusters de secciones censales con saldos negativos se ubican en zonas tipo $D$, es decir, caracterizadas por una alta presencia de extranjeros no europeos con bajo nivel educativo (zonas 9, 11 y 13), o próximas a éstas. Algo similar ocurre en el caso de la población no europea con alto nivel educativo, que retrocede en las zonas en donde predomina la población extranjera con bajo nivel educativo, como las secciones próximas a la zona degradada del Cabanyal-Canyamelar, o los barrios de Torrefiel-Poble Nou al norte, y Camí Real al sur. Por el contrario, la población extranjera con menor nivel educativo constituye el reverso de este modelo, saliendo del espacio central ya gentrificado y/o en vías de gentrificación como las zonas tipo A y B centrales, el área de Benimaclet-Sant Llorens, o las secciones censales del barrio de Cabanyal-Canyamelar contiguas al frente marítimo que está recibiendo población extranjera con un nivel educativo alto.

\section{Estabilidad del modelo territorial y tendencias de cambio}

A partir de la descripción anterior, resulta evidente que existe una asociación negativa entre los movimientos residenciales de algunos de los grupos sociodemográficos analizados; es decir, que la llegada de población de un perfil determinado se asocia a la salida mayoritaria de la población de otro grupo, y a la inversa. Teniendo esto en cuenta, el análisis cruzado de los espacios atractivos y de salida de los distintos colectivos nos permite caracterizar el patrón espacial de la movilidad de la población extranjera durante el periodo analizado:

1.- Existen algunas zonas de especialización socioresidencial en determinados colectivos de población extranjera en los que se está produciendo un refuerzo de esta especialización a través de la llegada de población de un perfil determinado, y la salida del resto. Estas zonas se están ampliando territorialmente sobre las secciones y barrios próximos. Son de dos tipos:

1.1. Zonas especializadas en población nacional de la Unión Europea con alto nivel educativo: Ocupan principalmente los espacios centrales de la ciudad, y se corresponden con las áreas que hemos caracterizado como de tipo B (zonas 2, 3 y 6). Estas zonas tienen saldos positivos de población inmigrante con este perfil, mientras que experimentan salidas netas de la población extranjera de países de fuera de la UE con bajo nivel educativo. La movilidad residencial está ampliando su extensión sobre los espacios contiguos, algunos de ellos también zonas residenciales de alta renta, pero aún con escaso nivel de población extranjera: barrios de Gran Vïa, Pla del Remei, Arrancapins y La Roqueta. 
1.2. Zonas especializadas en población de países de fuera de la UE con bajo nivel educativo: Estas áreas están reforzando su especialización con la llegada de nuevos habitantes del mismo perfil, y la salida de población cualificada: Nou Moles-Soternes-La Llum al oeste, y la Avenida del Puerto al este (zonas 9 y 13). Al sur de la primera, la dinámica de movilidad residencial está ampliando la zona segregada en los barrios de Vara de Quart, Safranar y la Fontsanta, y extendiéndose hasta Patraix, la Raiosa, y Camí Real.

2.- Por otra parte, las preferencias de movilidad residencial de la población extranjera con alto nivel educativo, principalmente de origen europeo, se han focalizado sobre algunos espacios urbanos que se han convertido en zonas atractivas para este tipo de población. Las principales zonas son las siguientes. Por un lado, el área de Nazaret-La Punta-Grao, ubicada en la parte litoral de las zonas 5 y 9, y la zona de Marxalenes-les Tendetes-Benicalap-Torrefiel-Tormos; que, probablemente gracias a nuevos proyectos de urbanización, están recibiendo población europea de alto nivel educativo. El tercer espacio en transformación, que recibe extranjeros tanto de dentro como de fuera de la UE con alto nivel educativo, se ubica en los barrios de En Corts y $\mathrm{Na}$ Rovella, próximos a la zona gentrificada de La Ciutat de les Arts y Penyarroja. Finalmente, un último clúster de este tipo es el barrio de El Cabanyal-Canyamelar, que, debido a la existencia de un área degradada ocupada por población europea de bajo nivel educativo (fundamentalmente rumana) y de un proyecto de renovación urbana apenas iniciado, recibe, por un lado, población extranjera de alto nivel educativo en el frente marítimo, mientras que este mismo grupo de población abandona la zona próxima al área degradada, y la población extranjera de bajo nivel educativo retrocede en el área intersticial.

3.- En este contexto de especialización residencial, la única zona en donde podemos constatar una dinámica de movilidad residencial que apunta a una cierta mezcla sociodemográfica es el clúster ubicado en los barrios de Mestalla-Exposición-La Xerea, en donde, en una zona de nivel de renta relativamente elevado, encontramos un saldo positivo de población extranjera de los tres tipos considerados.

Por tanto, y en relación con las cuestiones planteadas inicialmente, podemos concluir que la movilidad residencial de la población extranjera está contribuyendo, en líneas generales, a una consolidación de la estructura sociodemográfica anterior, al mismo tiempo que se detectan algunos cambios tendentes principalmente a ampliar territorialmente las áreas ya establecidas, al ubicarse nueva población del mismo perfil en secciones censales próximas a zonas ya especializadas. Estas zonas se ubican, en el caso de los extranjeros comunitarios de alto nivel educativo, en los espacios centrales de la ciudad; mientras que los extranjeros no comunitarios con bajo nivel educativo se localizan principalmente en la periferia urbana.

Existen, no obstante, algunas tendencias que contribuyen a modificar el patrón establecido, debidas principalmente a las pautas de comportamiento espacial de la población extranjera con mayor nivel educativo, que tiende a ubicarse en algunos espacios urbanos revitalizados por proyectos urbanísticos concretos o por procesos de renovación urbana más amplios. En este contexto, resultan especialmente interesante los procesos experimentados por el barrio de El Cabanyal-Canyamelar, en donde en un territorio limitado, en secciones censales contiguas, pueden observarse fenómenos de cambio residencial muy contrastados, indicativos de un intenso proceso de transformación residencial.

\section{CONCLUSIÓN}

Los microdatos de movilidad residencial de la población extranjera proporcionan una información útil para estudiar y analizar las dinámicas demográficas urbanas y sus implicaciones en la estructura territorial sociodemográfica de la ciudad. La aplicación de herramientas de estadística espacial ha permitido identificar cuáles de estas dinámicas constituyen una tendencia significativa desde un punto de vista estadístico.

En el caso estudiado, se ha podido concluir que, en la ciudad de Valencia, pese a que, tal y como afirman distintos autores, los niveles de segregación territorial son reducidos, existen desde el punto de vista residencial zonas preferentes para determinados grupos de población en función de su nacionalidad y perfil educativo y social, mientras que existen otras que, menos atractivas en términos netos, expulsan población de dichos perfiles.

Tal y como hemos mostrado en la tabla 2, durante el periodo post-crisis (2014-2017), la entrada de población extranjera en Valencia muestra, a diferencia de la española, un saldo neto positivo, lo que, unido a su elevada movilidad residencial intraurbana, hace que este grupo de población tenga un peso importante en la conformación de la estructura sociodemográfica de la ciudad. Aunque la entrada de población extranjera con nivel educativo bajo, indicativo de un menor nivel económico, es numéricamente importante, también lo es el número de salidas de este grupo de población, 
por lo que, en términos netos, es la población extranjera con mayor nivel educativo la que tiene un papel más importante a la hora de conformar la estructura urbana.

A este respecto, y en relación con las cuestiones planteadas inicialmente, podemos concluir que la movilidad residencial de la población extranjera está contribuyendo, en líneas generales, a la consolidación de la especialización territorial existente, detectándose algunos cambios tendentes principalmente a ampliar territorialmente las áreas ya establecidas, al ubicarse nueva población del mismo perfil en secciones censales próximas a las zonas ya especializadas. Estas tendencias están en la línea de lo observado en otras ciudades europeas, es decir, la tendencia a una creciente separación espacial entre las clases altas y bajas dentro del espacio urbano (Musterd et al., 2017; Hochstenbach y Musterd, 2018).

Por otro lado, las principales modificaciones del patrón territorial preexistente se deben a la emergencia de nuevos espacios atractivos para la población extranjera de nivel educativo más elevado en entornos específicos que han sido revitalizados por proyectos urbanísticos concretos o por procesos de renovación urbana más amplios. Se corrobora así otro aspecto característico de los modelos territoriales actuales de segregación urbana al que hacíamos referencia en la introducción: el creciente papel de la población extranjera de Europa Occidental en la formación de nuevos espacios segregados de clases altas que pueden dar lugar a procesos de gentrificación.

Los procesos descritos podrían producir, en una ciudad con -hasta el momento- niveles de segregación espacial no especialmente elevados, un cambio a medio plazo de la estructura existente, agravando las desigualdades territoriales y sociales. Sin embargo, esto está condicionado a los efectos que pueda tener la pandemia de COVID-19 sobre la movilidad residencial, en función tanto de su prolongación en el tiempo, como de la distinta intensidad de sus efectos contrapuestos sobre la dicotomía centralización/descentralización de los distintos grupos poblacionales.

\section{RECONOCIMIENTOS:}

La autora agradece la colaboración del Servicio de Estadística del Ayuntamiento de Valencia. Esta publicación es parte del proyecto de I+D+i PID2020/112734RB-C31, financiado/a por MCIN/ AEI/10.13039/501100011033/

\section{REFERENCIAS BIBLIOGRÁFICAS}

Ajuntament de Valencia. (2015). Estrategia de Desarrollo Urbano Sostenible e Integrado del barrio Cabanyal-Canyamelar-Cap de França. Valencia. Recuperado de https://www.valencia.es/edusi3c/ sites/default/files/docs/e.d.u.s.i._cabanyal-canyamelar-cap_franca_compressed.pdf

Andújar Llosa, A. (2017). Movilidad residencial y (re) composición social del espacio urbano en el municipio de Madrid. Papers: Revista de Sociología, 102(4), 761-792.

Bayona i Carrasco, J. (2007). La segregación residencial de la población extranjera en Barcelona: ¿una segregación fragmentada?. Scripta Nova: Revista Electrónica de Geografía y Ciencias Sociales, (11), 229-235. http://www.ub.edu/geocrit/sn/sn-235. htm

Bayona i Carrasco, J., y López Gay, A. (2011). Concentración, segregación y movilidad residencial de los extranjeros en Barcelona. Documents D’Anàlisi Geogràfica, 57(3), 381-412.

Bayona-i-Carrasco, J., Pujadas Rúbies, I. (2020). Las grandes áreas metropolitanas en España: del crecimiento y la expansión residencial al estancamiento poblacional. Documents d'Anàlisi Geogràfica, 66(1), 27-55.

Bayona-i-Carrasco, J.; Gil-Alonso, F.; Rubiales-Pérez, M. y Pujadas-Rúbies, I. (2018). New spatial mobility patterns in large Spanish cities: From the economic boom to the great recession. Applied Spatial Analysis and Policy, 11(2), 287-312.

Benassi, F; Iglesias-Pascual, R. y Salvati, L. (2020). Residential segregation and social diversification: Exploring spatial settlement patterns of foreign population in Southern European cities. Habitat International, 101, 102200. https:// www.sciencedirect.com/science/article/pii/ S0197397520300588

Domínguez Mujica, J., Díaz Hernández, R., y Parreño Castellano, J. M. (2010). Inmigración y ciudad en España: integración versus segregación socioterritoriales. Scripta Nova: Revista Electrónica de Geografía y Ciencias Sociales, XIV(331), 50. Recuperado de http://www.ub.edu/geocrit/sn/sn331/sn-331-50.htm

Duque-Calvache, R. (2017). Presentación. La relevancia social de la movilidad residencial. Papers 102(4), 537-541.

ESRI. (2021). Grouping Analysis. Recuperado de https:// 
desktop.arcgis.com/en/arcmap/10.3/tools/spatial-statistics-toolbox/grouping-analysis.htm

Florida. R., Rodríguez-Pose, A. y Storper, M. (2020). Cities in a Post-COVID World. Papers in Evolutionary Economic Geography, 1-23. doi 10.1177/00420980211018072

Galeano, J. y Bayona-i-Carrasco, J. (2018). Residential segregation and clustering dynamics of migrants in the metropolitan area of Barcelona: A demospatial analysis at the census tract level. Revue Quetelet, 6(1), 99-127. https://ojs.uclouvain.be/ index.php/Quetelet/article/view/18353

Hochstenbach, C. y Musterd, S. (2018). Gentrification and the suburbanization of poverty: changing urban geographies through boom and bust periods. Urban Geography. 39(1), 26-53. https://www.tandfonline.com/doi/full/10.1080/02723638.2016.1 276718

Iglesias Pascual, R. (2015). Segregación residencial, concepciones espaciales subjetivas y xenofobia en Sevilla. Determinando el umbral para la intervención social. Cuadernos Geográficos, 54(1), 230-256.

Iglesias Pascual, R. (2016). Espacio inducido y territorialización del discurso. Determinando el impacto socio-territorial del imaginario social sobre la inmigración en el Área Metropolitana de Sevilla. Documents d'Analisi Geografica, 62(2), 299-325.

Ilisei, R. y Salom, J. (2018). Urban Projects and Residential Segregation: A Case Study of the Cabanyal Neighborhood in Valencia (Spain). Urban Science, 2(4), 119. doi: 10.3390/urbansci2040119

López-Gay, A. (2014). Population growth and re-urbanization in Spanish inner cities: The role of internal migration and residential mobility. Revue Quetelet, 1 (2), 67-92

López-Gay, A.; Andújar-Llosa, A y Salvati, A. (2020). Residential Mobility, Gentrification and Neighborhood Change in Spanish Cities: A Post-Crisis Perspective. Spatial Demography, 8(3), 351-378.

Lloyd, C.D: (2010). Spatial Data Analysis. An Introduction for GIS Users. Oxford, Oxford University Press.

Malheiros, J. (2002). Ethnic-ities: residential patterns in the Northern European and Mediterranean Metropolises. Implications for Policy Design. International Journal of Population Geography, 8, 107-134. doi: 10.1002/ijpg.247

Massey, D. S. y Denton, N. A. (1985). Spatial assimila- tion as a socioeconomic outcome. American Sociological Review, 50(1), 94-106. https://www.jstor. org/stable/2095343?seq=1\#metadata_info_tab_ contents

Méndez del Valle, R. (2019). Ciudades en venta. Estrategias financieras y nuevo ciclo inmobiliario en España. Valencia, España: PUV, Col, Desarrollo Territorial, 21.

Musterd, S., Marcińczak, S., van Ham, M., y Tammaru, T. (2017). Socioeconomic segregation in European capital cities. Increasing separation between poor and rich. Urban Geography, 38(7), 1062-1083. https://www.tandfonline.com/doi/full/10.1080/ 02723638.2016.1228371

Nel.lo, O. (2004). ¿Cambio de siglo, cambio de ciclo?: Las grandes ciudades españolas en el umbral del siglo xxi. Ciudad y Territorio: Estudios Territoriales, 141-142, 523-542.

Ord, J.K. and A. Getis. (1995). Local Spatial Autocorrelation Statistics: Distributional Issues and an Application. Geographical Analysis, 27(4), 286-306. https://onlinelibrary.wiley.com/doi/10.1111/j.1538-4632.1995. tb00912.x

Pacione, M. (2009). Urban Geography. A global perspective, Routledge, Abingdon.

Palomares-Linares, I.; Feria, J.M; Susino, J. (2017). Medida y evolución de la movilidad residencial en las áreas metropolitanas españolas. Papers, 102(4), 545-575. https://www.raco.cat/index.php/Papers/article/download/v102-n4-palomares-feriasusino/419829

Portes, A. y Zhou, M. (1993). The new second generation: Segmented assimilation and its variants. The Annals of the American Academy of Political and Social Science, 530 (November), 74-96.

Rogers, A., y Hennig, S. (1999). The internal migration patterns of the foreign-born and native-born populations in the United States: $1975-80$ and 1985-90. International Migration Review, 33(2), 403-429.

Romero González, J; Salom-Carrasco, J.; Albertos Puebla, J. M; Pitarch-Garrido, M. D. y Melo Escrihuela, C. (2018). Trayectoria y recomposición metropolitana post-crisis en Valencia: ¿a la búsqueda de nuevos modelos?. Ciudad y Territorio. Estudios Territoriales, 50(198), 679- 696.

Rubiales, M.; Bayona, J. y Pujadas, I. (2012). Patrones espaciales de la segregación residencial en la Región Metropolitana de Barcelona. Pautas de 
segregación de los grupos altos. Scripta Nova, Revista Electrónica de Geografía y Ciencias Sociales, XVI(423). Recuperado de http://www.ub.edu/ geocrit/sn/sn-423.htm

Rubiales, M. (2020). Segregación en las metrópolis españolas 2001-2011. Un análisis con detalle territorial. Documents d'Analisi Geografica, 66(1), 83-105.

Sabater, A.; Galeano, J. y Domingo, A. (2013). La transformación de las comunidades mayoritarias y la formación y evolución de los enclaves étnicos residenciales en España. Migraciones, 34, 11-44.

Salom, J., y Fajardo, F. (2017). Cambios recientes en la estructura territorial sociodemográfica del área metropolitana de Valencia (2001-2011). Boletín de la Asociación de Geógrafos Españoles, (73). doi: 10.21138/bage.2412

Salom, J. y Pitarch, M. D. (2014). El Área Metropolitana de Valencia en la crisis. En Sánchez, J. L. y Albertos. J.M. (Eds.): Geografía de la crisis económica en España 539-563. Valencia, España: Universitat de València

Salom, J. y Pitarch, M. D. (2021). Cambios económicos, movilidad residencial y gentrificación en la ciudad de Valencia (2014-2017). Documents d'Anàlisi Geogràfica, 67(3), 521-560.

Sorando, D. y Leal, J. (2019). Distantes y desiguales: el declive de la mezcla social en Barcelona y Madrid. Revista Española de Investigaciones Sociológicas, 167, 125-148. http://www.reis.cis.es/REIS/PDF/ REIS_+167_081557488457491.pdf

Thiers-Quintana, J. y Gil-Alonso, F. (2020). Dinámicas residenciales de la inmigración latinoamericana en las metrópolis de Barcelona y Madrid: cambios de tendencias durante la expansión, la crisis y la poscrisis. Documents d'Anàlisi Geogràfica, 66(1), 57-82.

Torres, F. (director); Pérez, Y; Mut, E.; Giner, J. y Monsell, M. (2018). Hacia una ciudad intercultural. El vecindario inmigrante en València. 2006-2016. Estudiodiagnóstico de la inmigración en la ciudad de València y materiales para el diseño del Plan Municipal de Inmigración e Interculturalidad 2018-2022, Concejalía de Cooperación al Desarrollo y Migración. Valencia, España: Ayuntamiento De Valencia.

Viruela, R. y Torres, F. (2017). La movilidad internacional de los inmigrantes ecuatorianos y rumanos durante la crisis económica en España. Studi Emigrazione, LIV(206), 215-234.

\section{NOTAS}

1 Siguiendo a Palomares-Linares, Feria y Susino (2017), utilizamos el término de movilidad residencial para referirnos específicamente a los cambios residenciales intraurbanos (en este caso intramunicipales), y el de movilidad residencial y migratoria para referirnos al conjunto de todos los cambios de residencia, incluidos aquéllos que suponen un cambio de término municipal.

2 Ver la ubicación de los barrios en la figura 2.

3 La comparación realizada con los datos proporcionados por el INE nos confirma la necesidad de incluir en el cálculo las altas y, sobre todo, las bajas por otros motivos, ya que muchas de ellas son emigraciones definitivas que no se comunican. La incorporación de estos datos puede sobredimensionar ligeramente los movimientos de la población española, pero resulta imprescindible a la hora de evaluar los cambios experimentados por la población extranjera, sobre todo la nacional de países de fuera de la UE, que constituyen una parte muy importante del total.

4 Una estimación realizada por Torres et al. (2018, pág. 14) indica que, en 2016, había en el municipio 8.084 personas de nacionalidad no española nacidas en España, que quedarían fuera de cómputo en el caso de haber utilizado en el análisis el lugar de nacimiento en vez de la nacionalidad.

5 El certificado o título académico que se posee es un dato de inscripción obligatorio para el empadronamiento y debe recogerse adecuadamente, ya que, al margen del interés estadístico que pueda suponer, tiene una clara finalidad en los procesos electorales (artículo 26.2 de la LOREG), por lo que es objeto de distintos procesos de verificación por el INE. La calidad de la información sobre el nivel educativo incluida en el padrón de habitantes, que anteriormente no era muy buena, ha mejorado notablemente en los últimos años, debido a la actualización automática que se realiza a partir del registro de títulos del Ministerio de Educación, lo que permite un análisis pormenorizado de esta variable.

6 Se ha utilizado una técnica de interpolación espacial kriging, que, a través de la estimación de la autocorrelación espacial en función de la distancia y la orientación a partir de un punto dado, permite predecir la variación de los valores y detecta tendencias de variación locales (Lloyd, 2010). En 
este caso se ha aplicado kriging ordinario, con un modelo de semivariograma esférico, aplicando un tamaño de celda de $50 \mathrm{~m}$. de resolución y un radio de búsqueda variable de 12 puntos.

7 Esto no quiere decir que no haya una tendencia a la concentración, sino que no existen agrupaciones de secciones censales con una tendencia atractiva o repulsiva para este grupo de población estadística- mente significativa. Esta tendencia al agrupamiento puede producirse en el interior de la sección censal, por lo que no es posible detectarlo mediante un análisis que utiliza como unidad de estudio la sección censal. Esto es lo que ocurre con la población rumana, concentrada en una sección censal concreta de los Poblados Marítimos y con una tendencia a la dispersión aún insignificante estadísticamente. 